ARTICLE

\title{
Dietary lipids fuel GPX4-restricted enteritis resembling Crohn's disease
}

Lisa Mayr ${ }^{1,13}$, Felix Grabherr ${ }^{1} 13$, Julian Schwärzler ${ }^{1}$, Isabelle Reitmeier ${ }^{1}$, Felix Sommer (10 2 , Thomas Gehmacher ${ }^{1}$, Lukas Niederreiter ${ }^{1}$, Gui-Wei He ${ }^{3}$, Barbara Ruder ${ }^{3}$, Kai T. R. Kunz ${ }^{1}$, Piotr Tymoszuk (1) 4, Richard Hilbe ${ }^{4,5}$, David Haschka ${ }^{4}$, Clemens Feistritzer ${ }^{6}$, Romana R. Gerner ${ }^{1}$, Barbara Enrich¹, Nicole Przysiecki ${ }^{1,7}$, Markus Seifert ${ }^{4,5}$, Markus A. Keller (1) ${ }^{8}$, Georg Oberhuber ${ }^{9}$, Susanne Sprung ${ }^{10}$, Qitao Ran ${ }^{11}$, Robert Koch ${ }^{1}$, Maria Effenberger ${ }^{1}$, Ivan Tancevski ${ }^{4}$, Heinz Zoller ${ }^{1}$, Alexander R. Moschen (1) 1,7, Günter Weiss (1) 4,5, Christoph Becker ${ }^{3}$, Philip Rosenstiel ${ }^{2}$, Arthur Kaser ${ }^{12}$, Herbert Tilg ${ }^{1} \&$ Timon E. Adolph $^{1 凶}$

The increased incidence of inflammatory bowel disease (IBD) has become a global phenomenon that could be related to adoption of a Western life-style. Westernization of dietary habits is partly characterized by enrichment with the $\omega-6$ polyunsaturated fatty acid (PUFA) arachidonic acid (AA), which entails risk for developing IBD. Glutathione peroxidase 4 (GPX4) protects against lipid peroxidation (LPO) and cell death termed ferroptosis. We report that small intestinal epithelial cells (IECS) in Crohn's disease (CD) exhibit impaired GPX4 activity and signs of LPO. PUFAs and specifically AA trigger a cytokine response of IECs which is restricted by GPX4. While GPX4 does not control AA metabolism, cytokine production is governed by similar mechanisms as ferroptosis. A PUFA-enriched Western diet triggers focal granuloma-like neutrophilic enteritis in mice that lack one allele of Gpx4 in IECs. Our study identifies dietary PUFAs as a trigger of GPX4-restricted mucosal inflammation phenocopying aspects of human CD.

\footnotetext{
${ }^{1}$ Department of Internal Medicine I, Gastroenterology, Hepatology \& Endocrinology, Medical University of Innsbruck, Innsbruck, Austria. ${ }^{2}$ Institute of Clinical Molecular Biology, Christian-Albrechts-University Kiel and University Hospital Schleswig-Holstein, Kiel, Germany. ${ }^{3}$ Department of Medicine 1,

Gastroenterology, Pneumology and Endocrinology, University Medical Center Erlangen, Erlangen, Germany. ${ }^{4}$ Department of Internal Medicine II, Infectious Diseases, Immunology, Rheumatology, Pneumology, Medical University of Innsbruck, Innsbruck, Austria. ${ }^{5}$ Christian Doppler Laboratory for Iron Metabolism and Anemia Research, Medical University of Innsbruck, Innsbruck, Austria. ${ }^{6}$ Department of Internal Medicine V, Haematology and Oncology, Medical University of Innsbruck, Innsbruck, Austria. ${ }^{7}$ Christian Doppler Laboratory for Mucosal Immunology, Medical University of Innsbruck, Innsbruck, Austria. ${ }^{8}$ Institute of Human Genetics, Medical University of Innsbruck, Innsbruck, Austria. ${ }^{9}$ Pathology Department of Innsbruck Medical University Hospital, Innsbruck, Austria. ${ }^{10}$ Department of Pathology, Medical University of Innsbruck, Innsbruck, Austria. ${ }^{11}$ Department of Cell Systems and Anatomy, UT Health San Antonio, San Antonio, Texas, USA. ${ }^{12}$ Division of Gastroenterology and Hepatology, Department of Medicine, Addenbrooke's Hospital, University of Cambridge, Cambridge, UK. ${ }^{13}$ These authors contributed equally: Lisa Mayr and Felix Grabherr. ${ }^{凶}$ email: timon-erik.adolph@i-med.ac.at
} 
O xidation of biolipids, referred to as lipid peroxidation (LPO), is controlled by enzymatic (e.g. lipoxygenasemediated) and non-enzymatic (e.g. fenton-type) reactions that particularly affect polyunsaturated fatty acids (PUFAs) within biological membranes ${ }^{1-3}$. LPO impairs cellular functions partly by forming cytotoxic protein adducts or damage to cellular membranes ${ }^{4}$. Compensation is provided by glutathione peroxidase 4 (GPX4), the only selenoprotein that catalyzes the reduction of oxidized biolipids ${ }^{1,3,5}$. Deletion of both Gpx4 alleles in mice or pharmacologic GPX4 inhibition in cells induces a distinct regulated form of iron-dependent cell death termed ferroptosis ${ }^{1}$. Ferroptosis requires acyl-CoA synthetase long-chain family member 4 (ACSL4)-mediated membrane enrichment of the $\omega-6$ PUFA arachidonic acid (AA), which is prone to oxidation ${ }^{2,6,7}$. Deletion of both alleles of Gpx4 culminates in organ injury of the kidney, brain, and skin, which is conceivably elicited or modulated by immune responses ${ }^{3,8-11}$. While studies have identified key regulators of GPX4-restricted LPO and cellular demise ${ }^{1,2,6,12-14}$, mechanism(s) of concurrent inflammatory responses remain elusive.

Inflammatory bowel diseases (IBDs) and specifically Crohn's disease (CD) are characterized by chronic remittent intestinal inflammation that arises from complex interactions between environmental factors (e.g. diet) in a genetically susceptible host ${ }^{15}$. However, plausible examples to support this assumption remain scarce ${ }^{16-18}$. Notably, the increase in incidence of IBD parallels the increase in dietary intake of $\omega-6$ PUFAs such as AA, which is a major component of a Western diet and contained in meat and eggs ${ }^{19}$. Although AA intake entails a risk for developing IBD $^{20}$ and accumulates in the inflamed mucosa of IBD patients ${ }^{21}$, the impact of AA and PUFA metabolism on intestinal inflammation remains controversial ${ }^{22}$.

Given the genetic association between GPX4 and $\mathrm{CD}^{23}$ and reports of GPX4-restricted AA oxidation in biological membranes $^{2,6}$, we set out to study the role of intestinal epithelial GPX4 in controlling gut homeostasis ${ }^{24,25}$. We find that CD epithelium exhibits reduced GPX4 activity and features of LPO. In intestinal epithelial cells (IECs) with reduced GPX4 activity, PUFAs and specifically AA induce the release of interleukin 6 (IL-6) and chemokine (C-X-C motif) ligand 1 (CXCL1) which is governed by iron availability, lipoxygenase-mediated LPO and Acsl4. Mice that are exposed to a PUFA-enriched Western diet and that lack one Gpx4 allele in IECs $\left(G p x 4^{+/-I E C}\right)$, but not wild-type (WT) mice, display signs of epithelial LPO and focal neutrophilic enteritis with granuloma-like accumulation of inflammatory cells. Oral AA exposure evokes neutrophilic inflammation in the small intestine of iron-primed $G p x 4^{+/-I E C}$ mice. Enteritis in both models can be ameliorated by LPO scavenging. As such, our study exemplifies how PUFAs in a Western diet pose a risk for developing CD.

\section{Results}

Impaired epithelial GPX4 activity features CD. To investigate a role of reduced GPX4 activity and LPO in human IBD, we analyzed biopsy-derived IEC-enriched specimens from the lesional and nonlesional mucosa of CD and ulcerative colitis (UC) patients with active disease. Non-IBD patients who underwent screening colonoscopy and lacked demonstrable intestinal disease by endoscopic and histologic means served as healthy controls (HC). Clinical characteristics of this cohort are summarized in Table 1. IECs derived from the lesional small intestinal mucosa of CD patients exhibited decreased expression of GPX4, which was paralleled by decreased enzymatic activity (Fig. 1a-e and Supplementary Fig. 1A). In contrast, colonic GPX4 expression and activity in UC patients was indistinguishable from that in healthy controls (Fig. 1f, g), similar to GPX4 expression in colonic CD (Fig. 1f). In line with this, IECs of the lesional small intestinal mucosa of CD patients exhibited signs of LPO indicated by 4 -HNE adducts (Fig. 1h), which was similarly notable in small intestinal epithelial organoids retrieved from lesional mucosa of CD patients (Supplementary Fig. 1B).

PUFAs evoke an inflammatory response of Gpx4-deficient IECs. To analyze the role of GPX4 in IECs, we first generated Gpx $4^{-1-}$ small intestinal epithelial MODE-K cells by CRISPR Cas 9 editing of exon $1^{26}$, which induced IEC death and thus prevented further studies (Supplementary Fig. 1C, D). To assess the consequences of reduced (but not completely abrogated) GPX4 activity in IECs, we silenced MODE-K cells with Gpx4 small-interfering RNA (siGpx4). siGpx4 silencing impaired Gpx4 expression and enzymatic activity by $\sim 75 \%$ (Supplementary Fig. 1E-G). As oxidation of PUFAs and specifically AA is restricted by GPX $4^{2}$, we, in a first step, tested the impact of reduced GPX4 activity and AA exposure on intestinal epithelial LPO. Indeed, the $\omega-6$ PUFA AA deteriorated LPO in Gpx4-deficient IECs (Fig. 2a and Supplementary Fig. 1H). Importantly, AA induced the expression of IL-6 and CXCL1 in siGpx4 IECs, but not in control IECs (Fig. 2b-e). Similarly, $\omega-3$ and $\omega-6$ PUFAs, i.e. stearidonic acid (SDA), docosahexaenoic acid (DHA), eicosapentaenoic acid (EPA) and docosapentaenoic acid (DPA), induced LPO (Fig. 2f) and IL-6 and CXCL1 production (Fig. 2g, h) in siGpx4, but not in siCtrl IECs. The saturated long-chain fatty acid palmitic acid (PA) induced LPO, IL-6 and CXCL1 responses to a similar extent in siGpx4 and siCtrl IECs (Fig. 2f-h). Monounsaturated fatty acids such as palmitoleic acid (POA) and oleic acid (OA) did not impact LPO or cytokine production in siGpx4 IECs (Fig. 2f-h). Other proinflammatory stimuli such as TNFa or IL-1 $\beta$ evoked cytokine responses from siGpx4 IECs that were comparable to siCtrl IECs (Fig. 2i, j). As such, PUFAs particularly elicited LPO and a cytokine response in Gpx4-deficient IECs.

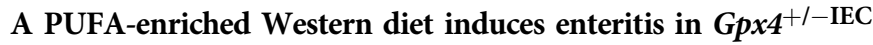
mice. Next, we set out to study the impact of PUFAs on intestinal inflammation in GPX4-deficient mice. We were unable to retrieve homozygous Gpx $4^{\text {flox/flox}}$; Villin-Cre $e^{+/-}\left(G p x 4^{-1-I E C}\right)$ mice as the offspring died in utero. However, $11 \%$ Gpx $4^{-/-I E C}$ pups were born when the diet of the mothers during gestation

Table 1 Patient characteristics.

\begin{tabular}{llll} 
& HC & CD & UC \\
\hline Total $N$ & 21 & 16 & 12 \\
male/female (\%) & $43 / 57$ & $50 / 50$ & $75 / 25$ \\
Age (years) & $51.62 \pm 12.1$ & $39.06 \pm 14.4$ & $40.38 \pm 13.7$ \\
Body mass index $\left(\mathrm{kg} / \mathrm{m}^{2}\right)$ & $28.56 \pm 6.17$ & $24.06 \pm 5.81$ & $23.70 \pm 3.48$ \\
MAYO Score & $\mathrm{N} / \mathrm{A}$ & $\mathrm{N} / \mathrm{A}$ & $4.75 \pm 0.69$ \\
Harvey-Bradshaw Index & $\mathrm{N} / \mathrm{A}$ & $4,67 \pm 0.52$ & $\mathrm{~N} / \mathrm{A}$ \\
CRP (mg/dl) & $\mathrm{N} / \mathrm{A}$ & $0.55 \pm 0.04$ & $0.59 \pm 0.14$ \\
\hline HC healthy control, CD Crohn's disease, UC ulcerative colitis, N patient numbers, CRP C-reactive protein. & & \\
\hline
\end{tabular}


a

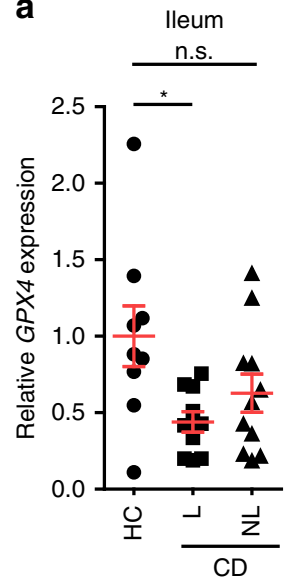

b

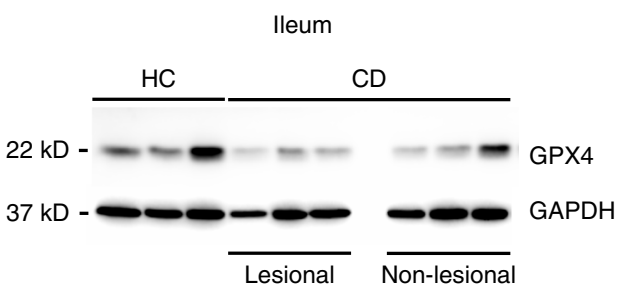

c

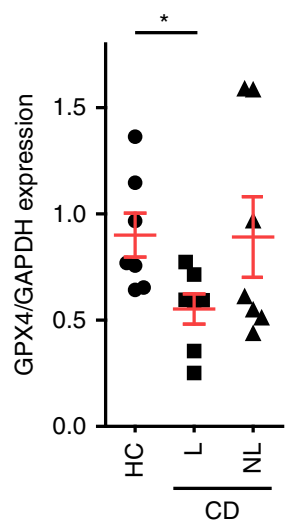

d

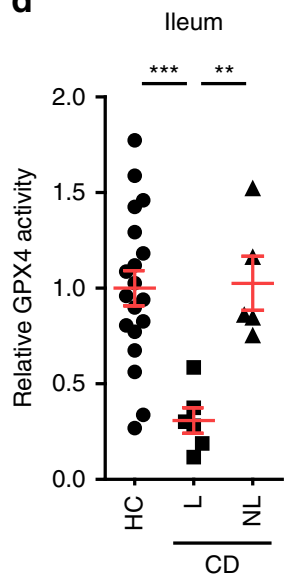

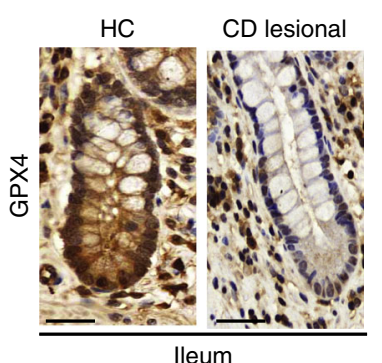

f

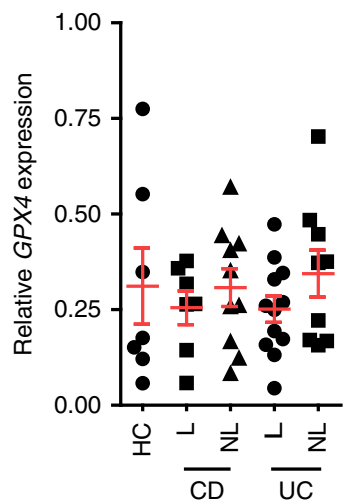

g

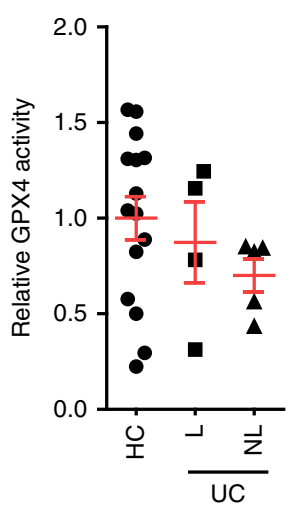

h

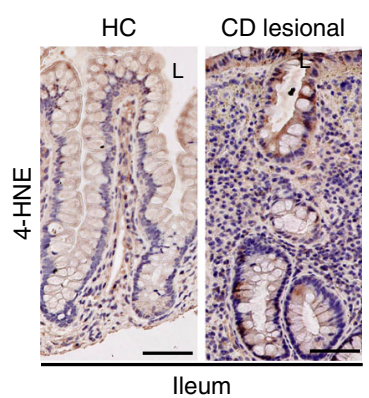

Fig. 1 Reduced GPX4 activity and LPO localize to IECs in CD patients. a Relative GPX4 expression in the macroscopically inflamed (lesional, L) and macroscopically non-inflamed (non-lesional, NL) small intestinal mucosa of CD patients determined by qPCR and compared to healthy controls (HC). Each dot represents one patient ( $n=9$ for HC, $n=11$ for CD-L, and $n=10$ for CD-NL). ${ }^{*}=0.0167$. b, c Representative GPX4 immunoblot of IEC-enriched fractions from biopsies taken from the ileum of $C D$ patients and $H C(\mathbf{b})$, with densitometry relative to GAPDH shown in (c). Each dot represents one patient ( $n=7$ patients per group). ${ }^{\star} P=0.0265$. $\mathbf{d}$ Relative GPX4 enzymatic activity of epithelial-enriched fractions derived from the lesional $(\mathrm{L})$ and nonlesional (NL) mucosa of the small intestine of CD patients as compared to HC. Each dot represents one patient $(n=19$ for HC, $n=6$ for CD-L, and $n=5$ for $\mathrm{CD}-\mathrm{NL}$ ). ${ }^{\star \star \star} P<0.001,{ }^{\star \star} P<0.01$. e Representative GPX4 immunoreactivity (brown) determined in the lesional small intestinal sections of CD patients as compared to HC $(n=7)$ ). f Relative GPX4 expression in the macroscopically inflamed (lesional, $\mathrm{L}$ ) and non-inflamed (non-lesional, NL) mucosa of the large intestine of CD patients and UC patients as compared to HC. Each dot represents one patient ( $n=7$ for HC, $n=9$ CD-L, $n=10$ for CD-NL, $n=12$ for UC-L and $n=7$ for UC-NL). $\mathbf{g}$ Quantification of GPX4 enzymatic activity in lesional and non-lesional IEC-enriched fractions from the large intestine of UC as compared to HC. Each dot represents one patient ( $n=15$ for HC, $n=4$ for UC-L, and $n=5$ for UC-NL). h 4-HNE immunoreactivity (brown) in HC and small intestinal lesional sections of CD patients, indicative for LPO. L, luminal-oriented side ( $n=3$ patients per group). Scale bars indicate $25 \mu \mathrm{m}$ (e) and $50 \mu \mathrm{m}(\mathbf{h})$. For panel (a), (c), (d), (f), and (g) data are presented as mean \pm SEM. One-way ANOVA with Bonferroni's multiple comparison test. Source data are provided as a Source Data file.

was supplemented with $\alpha$-tocopherol (Supplementary Table 1, Supplementary Fig. 2A, B). Gpx $x 4^{-I-I E C}$ pups from a-tocopherol treated mothers had lower weight at birth but regained weight at 7 weeks of age (Supplementary Fig. 2C-F), with a mucosa that was morphologically comparable to WT littermates (Supplementary Fig. 2G, H). For further studies, we utilised Gpxyflox/wt; Villin-Cre ${ }^{+l-}\left(G p x 4^{+/-I E C}\right)$ mice 27,28 , which specifically deleted one Gpx4 allele in the intestinal epithelium and resulted in a $\sim 50 \%$ reduction of mRNA and protein levels in small and large intestinal IECs (Supplementary Fig. 3A, B). Gpx $4^{+/-I E C}$ mice were viable, born at Mendelian ratio and exhibited a mucosal appearance indistinguishable from that of WT littermates (Fig. 3a and Supplementary Fig. 3C). LPO, IEC death and proliferation were comparable between $G p x 4^{+/-I E C}$ and WT mice (Supplementary Fig. 3D-H). Notably, Gp $x 4^{+/-I E C}$ mice were susceptible to colonic inflammation induced by dextran sodium sulfate (DSS) (Supplementary Fig. 3I-M), as previously observed in mice with myeloid-specific deletion of $G p \times 4^{29}$.

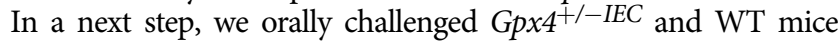
with a Western-style diet (ssniff TD88137) enriched with or without $10 \%$ fish oil (containing $\omega-3$ and $\omega-6$ PUFAs, Supplementary Table 2) for 3 months. As expected, WT mice were unaffected by a low-fat control diet (LFD), a Western diet (WD) or a PUFAenriched Western diet (PUFA WD) (Fig. 3b and Supplementary Fig. 4A). In contrast, a PUFA-enriched Western diet evoked patchy

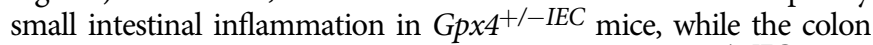
was unaffected. Specifically, PUFA WD-fed $G p x 4^{+/-I E C}$ mice displayed mucosal to submucosal infiltration of neutrophil granulocytes and mononuclear cells, crypt hyperplasia, epithelial injury and granuloma-like accumulation of inflammatory cells resembling some aspects of small intestinal CD (Fig. 3b-d and Supplementary Fig. 4B). Intestinal inflammation in PUFA WD-fed Gpx4+-IEC 


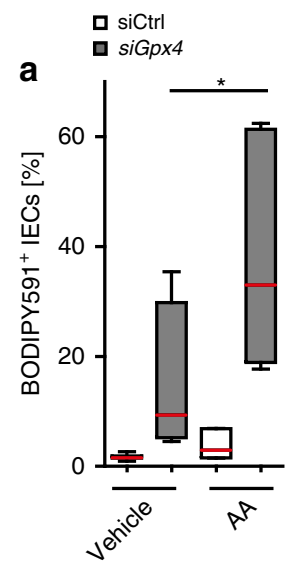

b

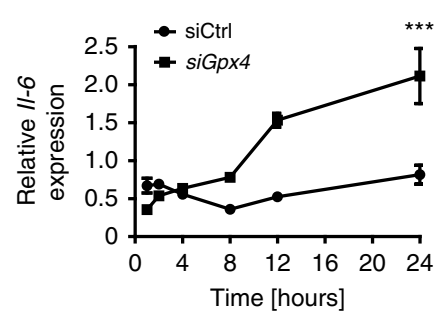

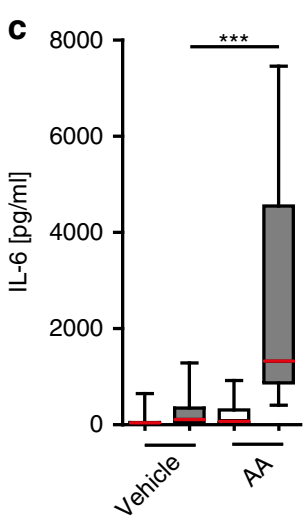

d

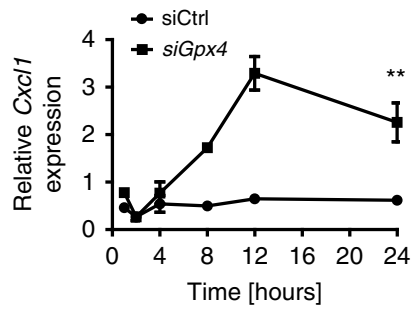

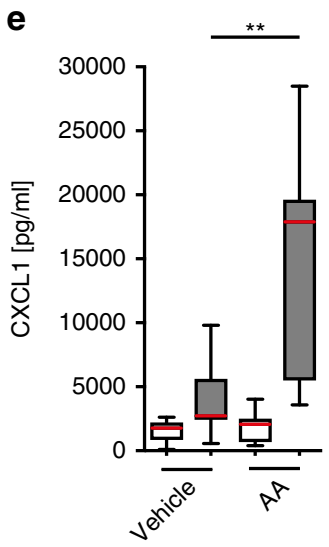

f

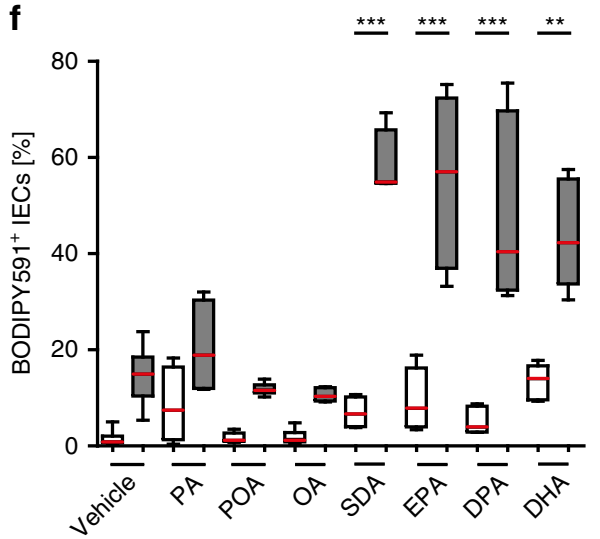

9

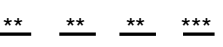

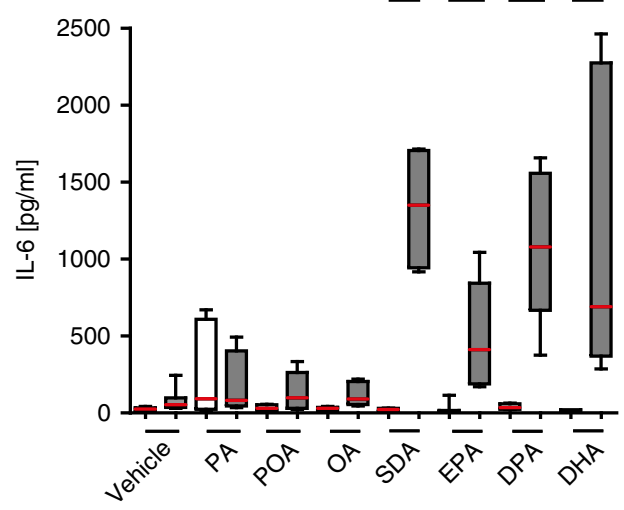

h

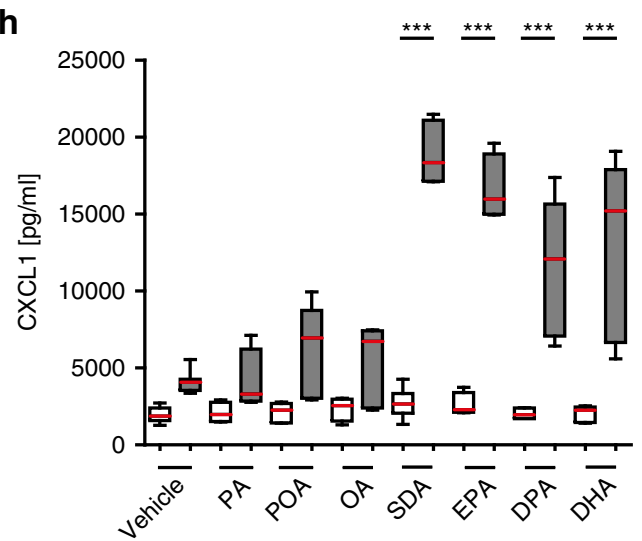

i

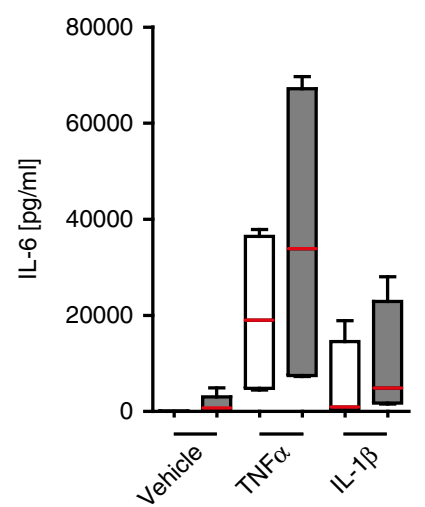

Fig. 2 PUFAs trigger epithelial LPO and an inflammatory response restricted by GPX4. a LPO quantification by flow cytometry of BODIPY581/591 C11 + labeled IECs stimulated with arachidonic acid (AA) for $24 \mathrm{~h}$ ( $n=6$ biologically independent experiments). ${ }^{\star} P=0.0183$. b, c Quantification of IL-6 expression from siGpx4 and siCtrl IECs over a course of AA stimulation determined by qPCR ( $n=4$ biologically independent experiments), ${ }^{\star \star *} P<0.001$ (b), and after $24 \mathrm{~h}$ by ELISA ( $n=12$ for vehicle and $n=24$ for AA biologically independent experiments). ${ }^{\star \star \star} P<0.001$ (c). d, e Quantification of CXCL1 expression from siGpx4 and siCtrl IECs over a course of AA stimulation determined by qPCR $\left(n=3\right.$ biologically independent experiments). ${ }^{\star \star} P=0.001$ (d) and after $24 \mathrm{~h}$ by ELISA ( $n=12$ for vehicle and $n=24$ for AA), ${ }^{\star \star} P=0.0031$ (e). $\mathbf{f}$ LPO quantification by flow cytometry of BODIPY581/591 C11+-labeled IECs stimulated with the saturated fatty acid palmitic acid (PA), monounsaturated fatty acids palmitoleic acid (POA) and oleic acid (OA) and polyunsaturated fatty acids stearidonic acid (SDA), eicosapentaenoic acid (EPA), docosapentaenoic acid (DPA) and docosahexaenoic acid (DHA) for $24 \mathrm{~h}$ ( $n=4-12$ biologically independent experiments) For all: ${ }^{\star \star \star} P<0.001$ and ${ }^{\star \star} P<0.01(\mathbf{g}, \mathbf{h})$. Quantification of IL-6 and CXCL1 expression from siGpx4 and siCtrl IECs stimulated with PA, POA, OA, SDA, EPA, DPA, and DHA for $24 \mathrm{~h}$ by ELISA ( $n=4-11$ biologically independent experiments). ${ }^{\star \star} P<0.001$ and ${ }^{\star \star} P<0.01$. i, $\mathbf{j}$ Quantification of IL-6 and CXCL1 production from siGpx4 and siCtrl IECs stimulated with TNF $\alpha$ or IL-1 $\beta$ for $24 \mathrm{~h}$ by ELISA ( $n=4$ biologically independent experiments). For panel (a), (c) and (e-j) data are presented as boxblot with median and interquartile range (25th and 75th). The whiskers represent minimal and maximal values. For panel (b) and (d) data presented as mean \pm SEM. For panel (a-j) to (j) one-way ANOVA with Bonferroni multiple comparison test or a Kruskal-Wallis test with Dunn's multiple comparison test was used with the exception of panel (b) and (d) for which a twoway ANOVA with Bonferroni post-hoc test was used and panel (f) and (g) for which an unpaired two-tailed Student's $t$ test was used. Source data are provided as a Source Data file. 
a

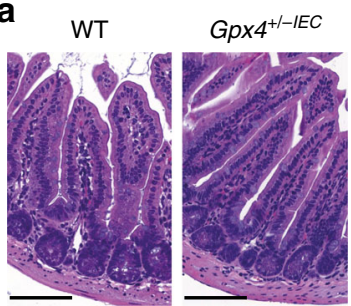

b
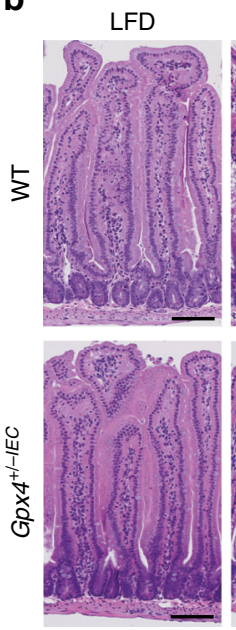

$\mathbf{f}$

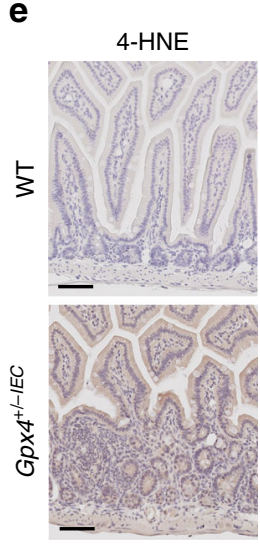

h

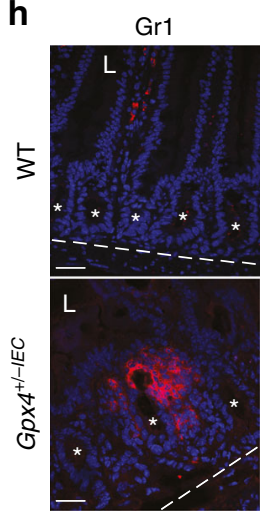

i

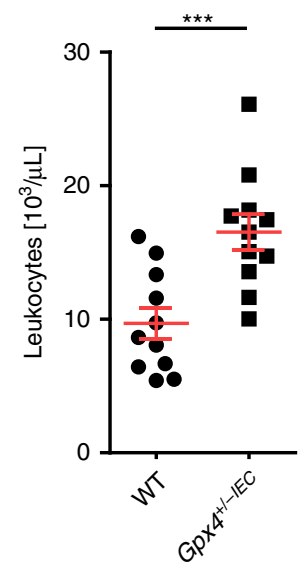

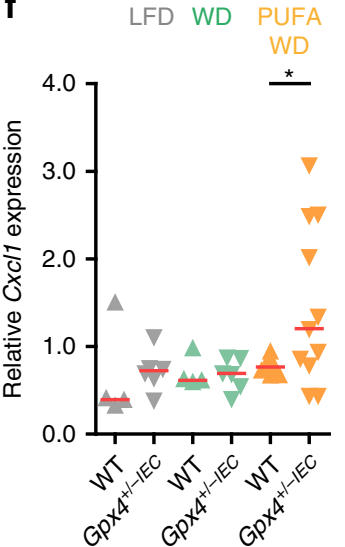

C

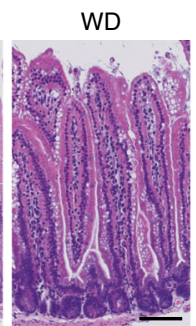

PUFA WD
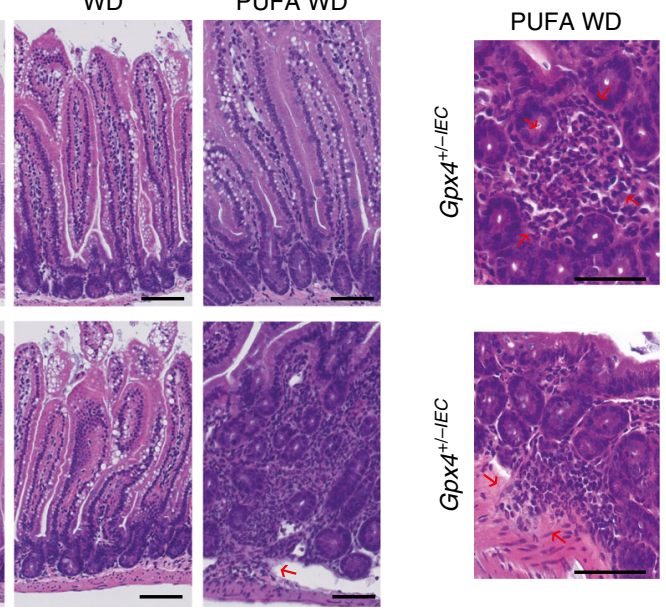

d

g

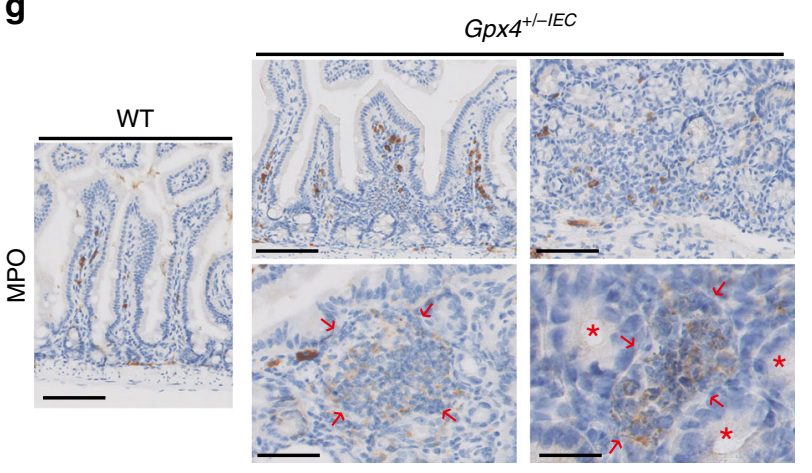

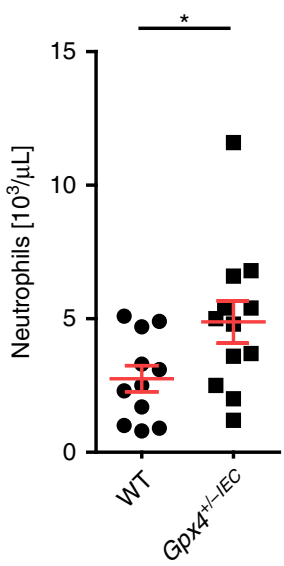

mice was characterized by signs of epithelial LPO (Fig. 3e), expression of Cxcl1 (Fig. 3f) and infiltration of $\mathrm{MPO}^{+}$and $\mathrm{GR}^{+}$ neutrophils (Fig. 3g, h). Gpx $4^{+/-I E C}$ mice also exhibited higher levels of circulating leukocytes and specifically neutrophil granulocytes in the blood (Fig. 3i). We observed a similar enteritis severity in male and female Gpx $x 4^{+/-I E C}$ mice (Supplementary Fig. 4C). Notably, a Western diet (rich in saturated fatty acids) did not induce intestinal inflammation in Gpx $x 4^{+/-I E C}$ mice (Fig. 3b, d and Supplementary Fig. 4D). Although PUFA stimulation (but not cytokines, bile acids or lipopolysaccharide) impaired epithelial GPX4 activity likely because of proteasomal degradation of GPX4 (Supplementary Fig. 5A-H) and Gpx4 deficiency induced ferroptosis of MODE-K
IECs to some extent (Supplementary Fig. 6A-I), we did not observe IEC death in Gpx $4^{+/-I E C}$ mice on a PUFA-enriched Western diet (Supplementary Fig. 6J-L). These data indicated that cell death was not a prerequisite of PUFA-induced and GPX4-restricted intestinal inflammation in $G p x 4^{+/-I E C}$ mice.

Cytokine production is driven by ferroptosis mechanisms. We focused on regulatory mechanisms that control AA-induced cytokine production because AA entails a risk for developing $\mathrm{IBD}^{20,21}$. As we noted that AA stimulation promoted LPO only in Gpx4-deficient IECs (Fig. 2a) and cellular iron availability and lipoxygenases (LOX) control LPO in Gpx4-deficient cells ${ }^{1,30}$, we 
Fig. 3 PUFA enrichment of a Western diet induces focal enteritis in Gpx4 $\mathbf{4}^{+/-I E C}$ mice. a Representative small intestinal H\&E images of Gpx4+/-IEC mice and WT littermates on a chow diet $(n=10$ mice per group). Scale bars indicate $100 \mu \mathrm{m}$. b Representative H\&E images of WT and Gpx4+/-IEC mice exposed to a low-fat diet (LFD), a Western diet (WD) or a PUFA-enriched WD (PUFA WD) for 3 months. Note that the PUFA WD evoked focal enteritis characterized by mono- and polymorphonuclear cell infiltration, crypt hyperplasia, and mucosal injury in Gpx4+/-IEC mice $(n=8$ mice for WT LFD, $n=9$

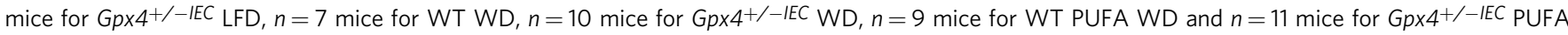
WD). Scale bars indicate $100 \mu \mathrm{m}$. c Granuloma-like accumulation of inflammatory cells and submucosal infiltration of inflammatory cells in Gpx4+/-IEC mice exposed to a PUFA-enriched WD for 3 months (red arrows) $(n=11)$. Scale bars indicate $100 \mu \mathrm{m}$. $\mathbf{d}$ Histology score of WT and Gpx4 ${ }^{+} /-I E C$ mice exposed to a low-fat diet (LFD), a Western diet (WD) or a PUFA-enriched WD (PUFA WD) for 3 months. Each dot indicates one experimental animal ( $n=$

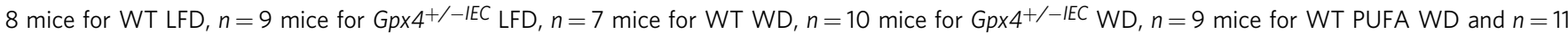
mice for Gpx4+/-IEC PUFA WD). Median shown, ${ }^{\star \star \star} P<0.001$. One-way ANOVA with Bonferroni's multiple comparison test. e Representative 4-HNE immunoreactivity (brown), indicative for LPO ( $n=5$ mice per group). Scale bars indicate $100 \mu \mathrm{m}$. $\mathbf{f}$ Relative $C x c / 1$ expression determined by qPCR ( $n=4$

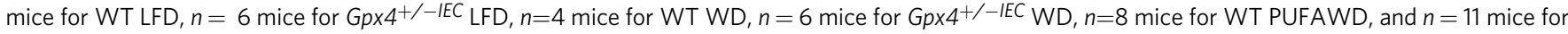
Gpx4+/-IEC PUFA WD). ${ }^{\star} P=0.0288$. One-way ANOVA with Bonferroni correction. $\mathbf{g}$ Representative images of $\mathrm{MPO}^{+}$cells in Gpx4+/-IEC and WT mice on a PUFA-enriched WD ( $n=5$ mice per group). The red arrows denote granuloma-like lesions with MPO ${ }^{+}$cells (brown) intermingled between crypts marked with asterisks. Scale bars indicate $50 \mu \mathrm{m}$ and $100 \mu \mathrm{m}$, respectively. $\mathbf{h}$ Representative confocal images of GR-1+ neutrophils (red) in Gpx4+/-IEC and WT mice on a PUFA-enriched WD ( $n=4$ mice per group). DAPI stained nuclei blue. Dashed line denotes basal membrane; $L$, luminal-oriented side. Asterisks denote crypt units. Scale bars indicate $50 \mu \mathrm{m}$. i Leukocyte count and neutrophil granulocyte count from whole blood samples of Gpx4 ${ }^{+/-I E C}$ and WT mice after a 3-month PUFA-enriched WD (Left panel $n=11$ mice, right panel $n=11$ mice for WT and $n=12$ mice for $G p \times 4^{+/-I E C}$ ). ${ }^{\star} P=0.0361$, ${ }^{\star \star \star} P<$ 0.001. Unpaired two-tailed Student's $t$ test. Source data are provided as a Source Data file.

analyzed the impact of iron and LPO on GPX4-restricted cytokine production. Ferric iron promoted LPO (Fig. 4a) and AAinduced cytokine production in siGpx4 IECs (Fig. 4b, c), despite a reduced iron uptake that could not be explained by differential regulation of iron transporters (Supplementary Fig. 7A-G). Vice versa, iron chelation with deferoxamine (DFO) reduced IL-6 and CXCL1 responses of siGpx4 IECs upon AA stimulation (Fig. 4d, e). Moreover, AA-induced cytokine responses were ameliorated by the LPO scavenger ferrostatin-1 and $a$-tocopherol (Fig. $4 \mathrm{f}-\mathrm{h}$ ). Similarly, inhibition of LOX15 ameliorated AA-induced LPO (Supplementary Fig. 7H) and cytokine production of siGpx4 IECs (Fig. 4i, j). Co-silencing of Alox15 (or Alox12) also ameliorated the cytokine response of siGpx4 IECs after AA stimulation (Fig. $4 \mathrm{k}$ and Supplementary Fig. 7I, J). The non-selective COX1/2 inhibitor piroxicam abolished neither CXCL1 production nor LPO (Supplementary Fig. 7K, L). Collectively, these data demonstrate that iron availability promoted PUFA-induced LPO and cytokine production in IECs with reduced GPX4 activity, which was reversed by a-tocopherol.

AA and ferric maltol induce enteritis in $G p x 4^{+/-I E C}$ mice. These findings led us to study the direct impact of AA and ferric iron on intestinal inflammation. Indeed, intestinal epithelial organoids from $G p x 4^{+/-I E C}$ mice, but not WT controls, displayed signs of LPO and increased Cxcl1 expression on AA and ferric iron exposure (Fig. 5a, b and Supplementary Fig. 8a), while unstimulated $G p x 4^{+/-I E C}$ organoids were indistinguishable from WT organoids (Supplementary Fig. 8B-G). Next, we orally challenged 8 -week old WT and Gpx $4^{+/-I E C}$ mice with AA once daily for five consecutive days complementary to the standard chow diet in addition to iron supplementation with ferric maltol (Fig. 5c), which is approved for treatment of iron-deficiency anemia in $\mathrm{IBD}^{31}$. We used this model as it may reflect a daily PUFA and iron challenge in humans on a meat-enriched Western $\operatorname{diet}^{19}$. WT mice were unaffected by oral AA and ferric maltol exposure (Fig. 5d, e). In contrast, Gpx $4^{+/-I E C}$ mice exhibited inflammation in the intestine when exposed to AA and ferric maltol (Fig. 5d, e). More specifically, acute inflammation in $G p x 4^{+/-I E C}$ mice was characterized by neutrophil infiltration in the proximal small intestine (Fig. 5d, e), the locale of PUFA and iron absorption ${ }^{32,33}$, as corroborated by flow cytometry of GR1 ${ }^{+}$neutrophils (Fig. $5 f$ ) and immuno-labeling of $\mathrm{MPO}^{+}$cells (Supplementary Fig. $8 \mathrm{H}$ ). Neutrophilic infiltration was paralleled by signs of epithelial LPO (Fig. 5g) and increased
Cxcl1 expression (Fig. 5h). Notably, ferric maltol or AA exposure alone did not evoke neutrophilic inflammation in $G p x 4^{+/-I E C}$ mice (Fig. 5e). The abundance of other mucosal innate and adaptive immune cells remained comparable between WT and $G p \times 4^{+/-I E C}$ mice exposed to AA and ferric maltol (Supplementary Fig. 9A-H) and we did not note colonic inflammation (Supplementary Fig. 10A). These data demonstrated that intestinal epithelial GPX4 restrained neutrophilic small intestinal inflammation induced by AA and ferric maltol. In line with a critical role of LPO in inflammation, $\alpha$-tocopherol treatment protected against PUFA-induced LPO and cytokine production (Fig. 4f-h) and neutrophilic infiltration in Gpx $4^{+/-I E C}$ mice challenged with AA and ferric maltol (Fig. 5i). Similarly, a-tocopherol, as well as liproxstatin-1 treatment, protected against enteritis in $G p \times 4^{+/-I E C}$ mice induced by a PUFA WD (Fig. 5j, k), which was associated with reduced signs of LPO and neutrophil infiltration (Supplementary Fig. 10B-D).

Cytokine production is governed by ACSL4. To further explore how AA may instigate cytokine production, we used liquid chromatography tandem mass spectrometry (LC-MS/MS) to investigate the AA metabolite profile of siGpx4 IECs as compared to that of controls. AA may be metabolized by cyclooxygenases (COX), lipoxygenases (LOX) and cytochrome P450 enzymes to bioactive lipid mediators, which occurred to a similar extent in AA-stimulated siGpx4 and siCtrl IECs (Fig. 6a-c, Supplementary Table 3). In line with this, Lox and Cox expression was comparable between siCtrl and siGpx4 IECs (Supplementary Fig. 11A). As Acsl4 is required for IEC ferroptosis (Supplementary Fig. 6I) $)^{2,6}$, we next hypothesized that AA-induced inflammation in siGpx4 IECs required ACSL4. Indeed, Acsl4 deletion abolished AA-induced IL-6 and CXCL1 production in siGpx4 IECs (Fig. 6d, e).

Next, we sought to understand how ACSL4 controlled AAinduced cytokine responses. Notably, Acsl4 deletion in siGpx4 IECs did not protect against LPO after AA exposure (Supplementary Fig. 11B). These data indicated that ACSL4 controlled AA-induced cytokine production using a distinct mechanism that was independent of LPO. To explore a role of ACSL4 in AA metabolism, we analyzed the metabolite profile in $A c s l 4^{-/-}$IECs by means of LC-MS/MS (Fig. 6a-c). Indeed, Acsl4 ${ }^{-/}$IECs exhibited a decreased abundance of LOX and COX metabolites after AA stimulation in siCtrl and siGpx4 IECs (Fig. 6a, b). 
a

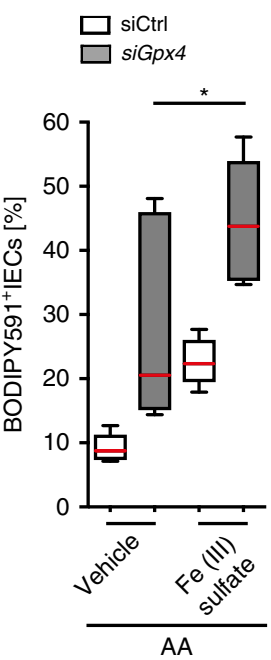

f

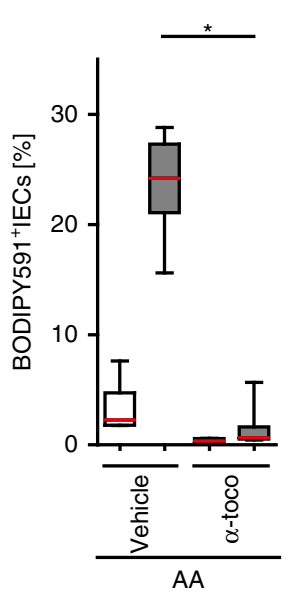

b

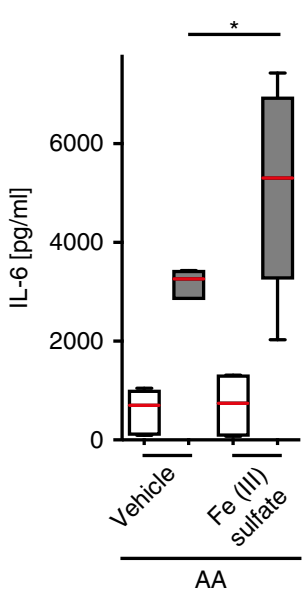

g

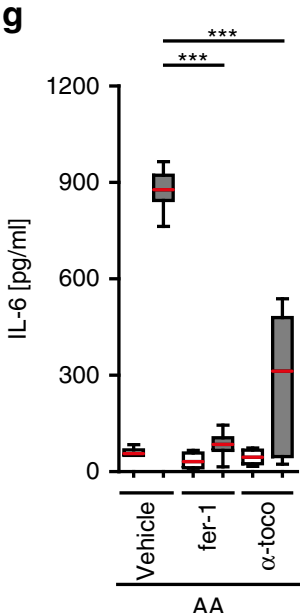

c

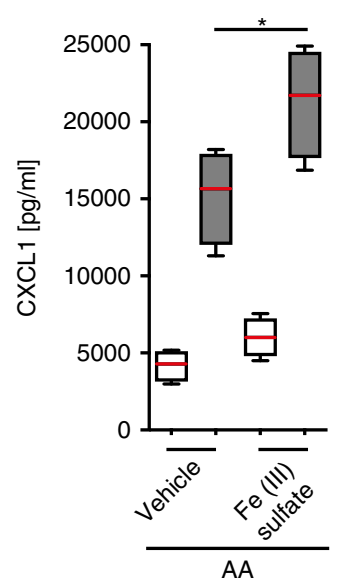

h

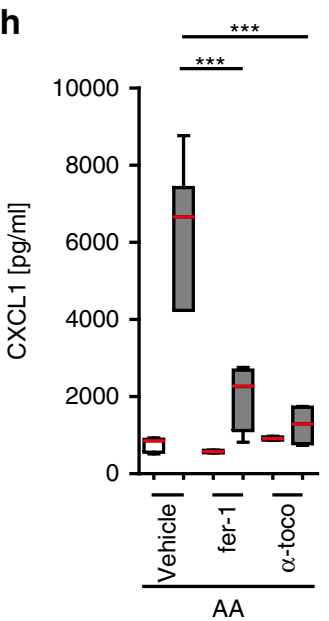

d
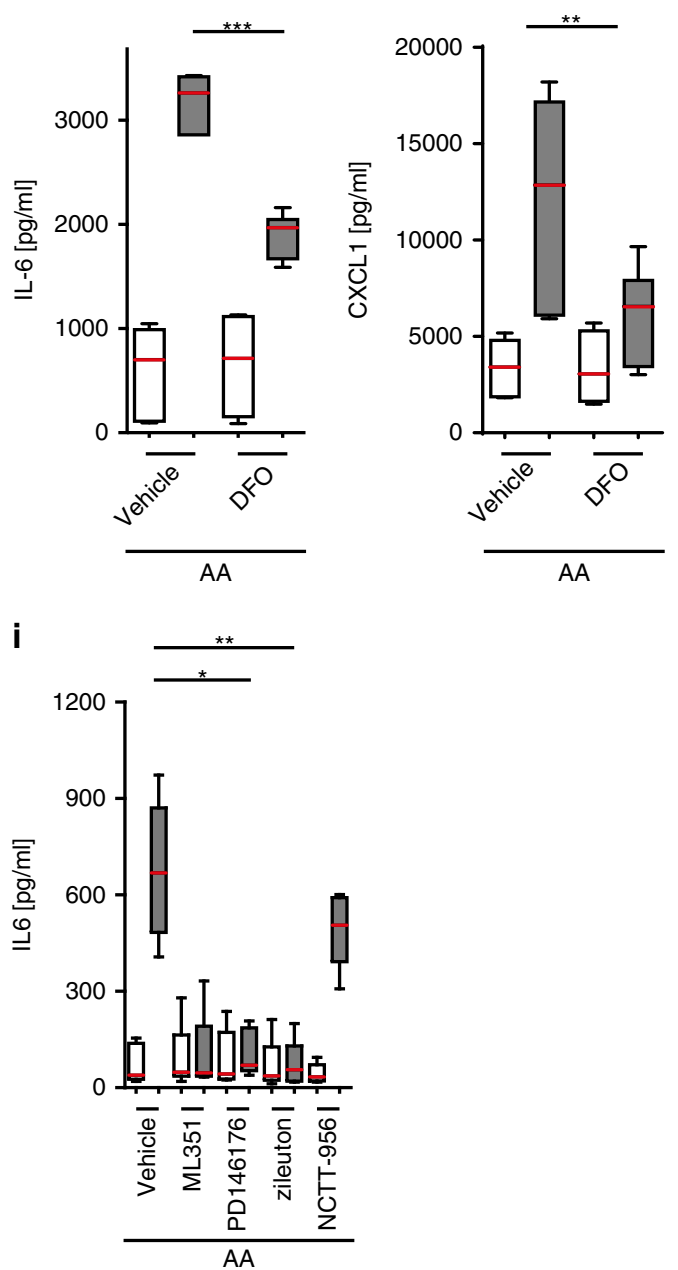
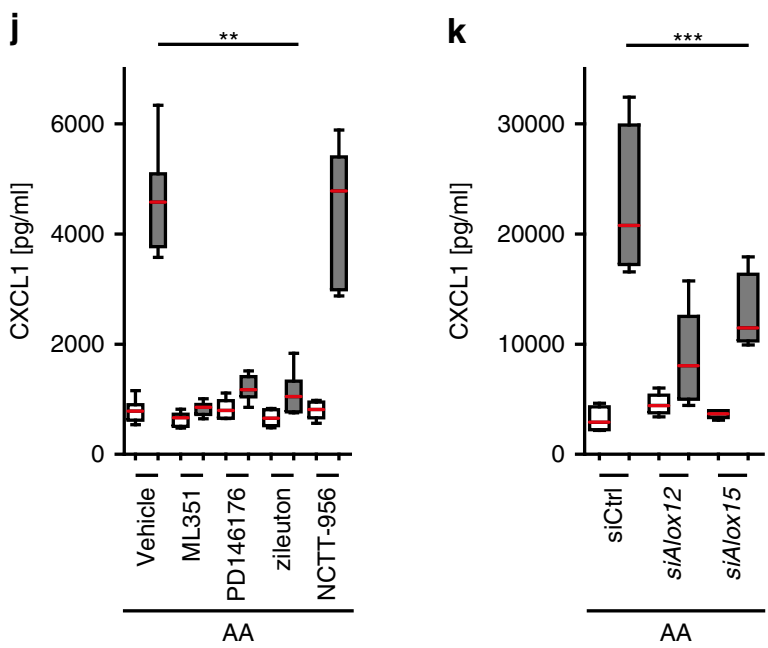

However, none of the abundant LOX and COX lipid mediators (i.e. 5-HETE, a 15-HETE precursor or PGE2) was able to promote the production of IL-6 or CXCL1 in our model (Supplementary Fig. 11C-H). These data suggested that modulation of LOX and COX metabolism by ACSL4 did not affect the inflammatory tone. However, we noted that P450 metabolites of AA (i.e. epoxyeicosatrienoic acids or 'EETs') were increasingly accumulating in
Acsl4 ${ }^{-/}$IECs after AA stimulation (Fig. 6c). A combination of EETs ameliorated AA-induced IL-6 and CXCL1 production in siGpx4 IECs (Fig. 6f, g), likely due to their anti-inflammatory effect that suppressed NF- $\mathrm{KB}^{34}$. In line with this, we noted that $\mathrm{AA}$ induced activation of NF- $\mathrm{\kappa B}$ p65 in siGpx4 IECs (Supplementary Fig. 11I, J), and NF-kB inhibition with BAY11-7082 or MG132 abolished IL-6 production in siGpx4 IECs independent of LPO 
Fig. 4 Iron availability, lipoxygenases and LPO control PUFA-induced cytokine production. a LPO quantification after $24 \mathrm{~h}$ stimulation with AA and ferric iron ( $5 \mu \mathrm{M} \mathrm{Fe}$ (III) sulfate) or vehicle ( $n=6$ biologically independent experiments). ${ }^{\star} P=0.0234$. b, $\mathbf{c}$ Quantification of IL- 6 and CXCL1 in the supernatant from siGpx4 and siCtrl IECs stimulated with AA and ferric iron or vehicle for $24 \mathrm{~h}(n=4$ biologically independent experiments).* $P=0.0479$ for $(\mathbf{b})$ and ${ }^{*} \mathrm{P}=0.0259$ for (c). d, e Quantification of IL-6 and CXCL1 in the supernatant from siGpx4 and siCtrl IECs stimulated with AA and deferoxamine (DFO) or vehicle ( $n=6$ biologically independent experiments). ${ }^{\star \star \star} P<0.001$ for $(\mathbf{d})$ and $(\mathbf{e})$. $\mathbf{f}$ LPO quantification by flow cytometry of BODIPY581/591 C11 ${ }^{+}-$labeled IECs after $24 \mathrm{~h}$ stimulation with AA and $\alpha$-tocopherol ( $\alpha$-toco) or vehicle $\left(n=6\right.$ biologically independent experiments). ${ }^{*} P=0.0199 . \mathbf{g}$, $\mathbf{h}$ Quantification of indicated cytokines in the supernatant from siGpx4 and siCtrl IECs after $24 \mathrm{~h} \mathrm{AA}$ stimulation co-treated with LPO scavengers ( $n=6$ biologically independent experiments in $(\mathbf{g})$ and $n=4$ biologically independent experiments in $(\mathbf{h})$ ). ${ }^{\star \star \star} P<0.001$. $\mathbf{i}, \mathbf{j}$ Cytokine quantification in the supernatant of siGpx4 and siCtrl IECs after $24 \mathrm{~h} \mathrm{AA}$ stimulation and treatment with selective LOX inhibitors $(n=5$ for vehicle and $n=3$ for indicated inhibitors; biologically independent experiments). ${ }^{\star} P<0.05$, ${ }^{\star \star} P<0.01,{ }^{\star \star \star} P<0.001$. $\mathbf{k}$ Quantification of CXCL1 in the supernatant from siGpx4 and siAlox12 or siAlox15 co-silenced IECs stimulated with AA $(20 \mu \mathrm{M})$ for $24 \mathrm{~h}\left(n=4\right.$ biologically independent experiments). ${ }^{\star \star \star} P<0.001$. Data are presented as boxblot with median and interquartile range (25th and 75th). The whiskers represent minimal and maximal values. One-way ANOVA with Bonferroni's multiple comparison test or Kruskal-Wallis Test with Dunn's multiple comparison test was used. Source data are provided as a Source Data file.

(Supplementary Fig. 11K, L). As such, Acsl4 deletion may limit AA-induced cytokine responses by modulating AA metabolism ${ }^{34}$, while GPX4 controlled LPO.

\section{Discussion}

Westernization of dietary habits, partially characterized by enrichment with PUFAs ${ }^{35,36}$, paralleled the increased IBD incidence $^{19}$. Previous observations associated PUFA uptake and mucosal AA accumulation with the risk of developing IBD $20,21,37$. Large prospective clinical trials in $\mathrm{CD}$ patients (and patients without IBD) indicated that PUFA supplementation may cause gastrointestinal side effects (e.g. diarrhea), indicative for disturbed intestinal homeostasis ${ }^{38,39}$. In contrast, dietary restriction (e.g. by an elemental diet) ameliorates the course of $\mathrm{CD}^{40,41}$. These reports and other studies ${ }^{15}$ indicate that dietary cues impact the risk of developing $\mathrm{CD}$ and affect the natural history of disease. However, a direct link between PUFA uptake and intestinal inflammation remained elusive. Our study establishes that dietary-derived PUFAs trigger neutrophilic inflammation in the small intestine resembling some aspects of human CD.

IECs have the delicate task of maintaining a physical and immunological line of defense to protect the host against a potentially hostile environment. At the same time IECs must continue to allow uptake of essential nutrients such as long-chain fatty acids ${ }^{42}$. A hypoxic milieu and exposure to luminal noxae may specifically require GPX4 activity in IECs to protect against cellular LPO, a critical condition that determines cell fate ${ }^{1}$. While substantial advances have shaped our understanding of ferroptosis $^{1}$, GPX4-restricted immunologic responses remain poorly explored despite reports of inflammatory tissue injury in GPX4deficient animals $9,11,27$. We report that IECs with reduced GPX4 activity respond to non-toxic dietary PUFA exposure with an inflammatory response involving IL-6 and CXCL1. PUFAinduced CXCL1 production of siGpx4 IECs was comparable to CXCL1 production induced by TNFa stimulation. In contrast IL6 production was far less pronounced in PUFA-stimulated siGpx4 IECs, when compared to TNFa stimulation. Future studies will delineate the relevance of both cytokines as driver of mucosal inflammation in our model. Notably, exposing $\omega-3$ PUFAs such as DHA (which is thought to exert anti-inflammatory effects ${ }^{43}$ ) elicited cytokine production similar to AA (which is thought to exert inflammatory effects) only in Gpx4-deficient IECs. As such it appears that the availability (rather than the positioning) of double bonds within PUFAs define their propensity to fuel LPO and an inflammatory response. These findings led us to explore the consequences of PUFA exposure on IECs with impaired GPX4 activity in the intestine. We generated mice with IECspecific deletion of one $G p x 4$ allele $\left(G p x 4^{+/-I E C}\right)$, which is a valuable tool for modeling the effects of reduced, but not completely abrogated epithelial GPX4 activity as observed in patients with small intestinal CD. Indeed, a PUFA-enriched WD containing $10 \%$ fish oil (with $\omega-3$ and $\omega-6$ PUFAs) evoked focal neutrophilic enteritis in male and female $G p x 4^{+/-I E C}$ mice that was characterized by granuloma-like mucosal to submucosal accumulation of inflammatory cells and expression of the IL-8 homologue CXcl1. As such, our findings represent first evidence that non-toxic dietary lipids trigger focal enteritis in a genetically susceptible host resembling some aspects of human small intestinal CD. Future studies are warranted to explore the immune-phenotype in more detail. Notably, a WD rich in saturated fatty acids (without addition of fish oil) did not induce intestinal inflammation in Gpx $4^{+/-I E C}$ mice, indicating that PUFA supplementation specifically elicited the inflammatory phenotype.

We did not note colonic intestinal inflammation in PUFA WD- or AA/FM-exposed Gpx4 $4^{+/-I E C}$ mice, suggesting that environmental cues may determine disease localization. We speculate that PUFAs and iron are specifically absorbed in the small intestine $32,33,44$, which could be one explanation for small intestinal disease localization and a requirement for GPX4 activity in the small intestine. In line with a small intestinal phenotype in mice, we specifically noted reduced epithelial GPX4 activity in active small intestinal $\mathrm{CD}$. In contrast, we did not note altered GPX4 activity in colonic CD or UC.

Of note, fish oil supplementation has been tested in the maintenance of remission in CD patients with mixed results. More specifically, fish oil supplementation had favorable effects on maintenance of remission in some patients ${ }^{45}$, while larger studies observed no beneficial effect but worsening of gastrointestinal symptoms (e.g. diarrhea) ${ }^{45-47}$. This observation indicates that dietary PUFA exposure impacts the course of CD. Similarly, PUFA supplementation induces gastrointestinal symptoms (diarrhea, abdominal pain and nausea) in patients without $\mathrm{IBD}^{39}$. Which factors define a beneficial or detrimental response on PUFA challenge (e.g. fish oil or other sources such as fish, meat and eggs) in CD (or healthy) patients is unknown. Our data indicate that PUFAs trigger GPX4-restricted mucosal inflammation resembling some aspects of human CD.

Ferroptosis is fundamentally controlled by lipoxygenasedriven LPO, which is limited by GPX4 and driven by cellular iron availability ${ }^{1}$. Similarly, we found that PUFA exposure induced LPO and cytokine production of siGpx4 IECs, while pharmacologic iron chelation with DFO, inhibition of lipoxygenases and LPO scavenging with a-tocopherol ameliorated this phenotype. Genetic deletion of Acsl4 (which is required for ferroptosis ${ }^{2,6}$ ), abrogated PUFA-induced cytokine production in IECs with reduced GPX4 activity. While GPX4 did not control AA metabolism, we found that ACSL4 limited the generation of anti-inflammatory AA metabolites (epoxyeicosatrienoic acids or 'EETs'), which have been demonstrated to inhibit NF- $\kappa B$ 
a

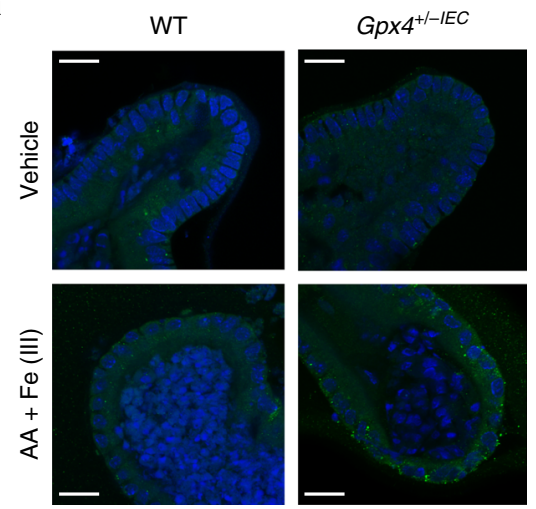

b

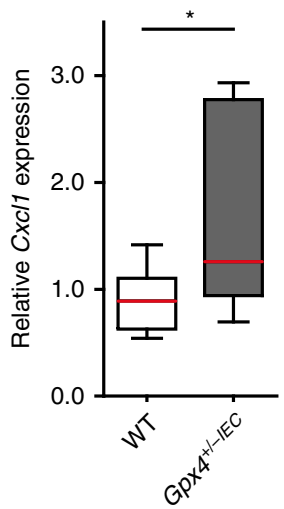

C

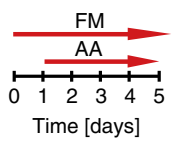

d WT

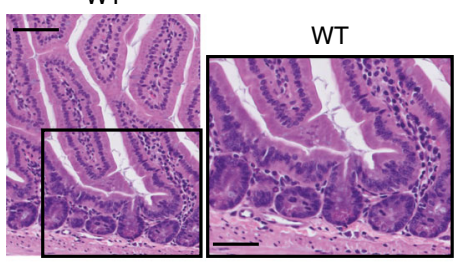

$G p \times 4^{+/-I E C}$

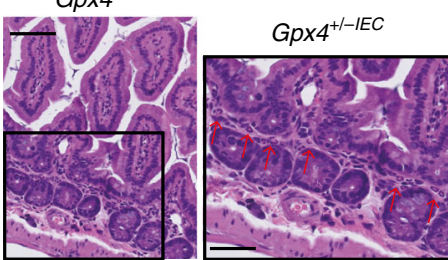

e

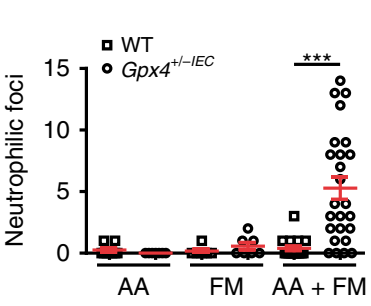

f

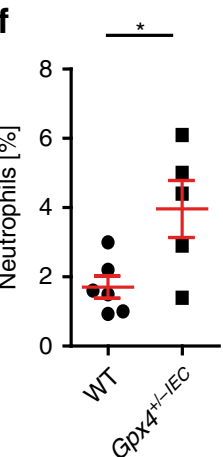

g

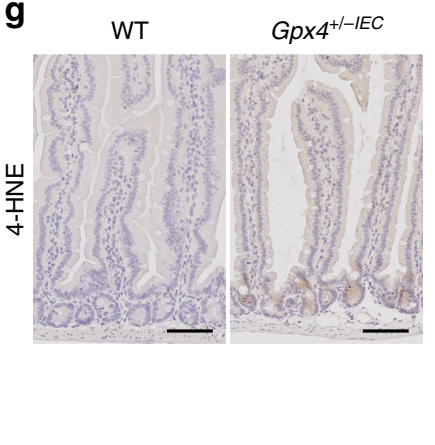

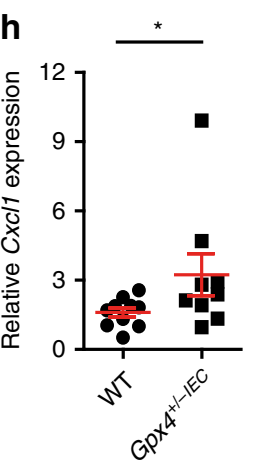

i j
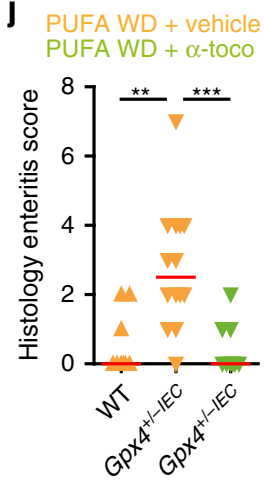

$\mathbf{k}$

PUFA WD + vehicle PUFA WD + liprox-1

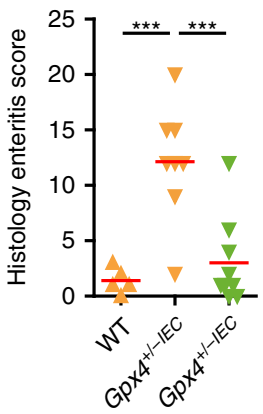

activity $^{34}$. Indeed, a combination of EETs, but not a single EET alone, reduced AA-induced cytokine production similar to pharmacologic inhibition of NF- $\kappa$ B. These data suggest that GPX4-restricted LPO and ACSL4-controlled AA metabolism converge on NF- $\kappa \mathrm{B}$-mediated transcription of inflammatory cytokines specifically in GPX4-deficient IECs. As such, the same mecha-

nisms (i.e. iron availability, LOX-mediated LPO and ACSL4) that control ferroptosis ${ }^{1}$ also control PUFA-induced cytokine production in siGpx4 IECs. These findings lay the foundation for understanding dietary lipid-induced intestinal inflammation. Indeed, AA and ferric maltol (approved for the treatment of iron-deficiency anemia in $\mathrm{IBD}^{31}$ ) triggered epithelial LPO, Cxcl1 expression and small intestinal neutrophilic infiltration in $G p \times 4^{+/-I E C}$ mice. Similarly, Gp $x 4^{+/-I E C}$ mice exposed to a PUFA WD exhibited increased LPO and neutrophilic inflammation, and LPO scavenging with a-tocopherol ameliorated enteritis in both models. These findings indicate that GPX4 protects against epithelial LPO, which sets a threshold for intestinal inflammation triggered by dietary lipids. Although LPO is a key feature of ferroptosis ${ }^{1}$, cell death was not a prerequisite for intestinal inflammation indicated by a lack of epithelial TUNEL labeling in Gpx $4^{+/-I E C}$ mice. One reason for this may be that one Gpx4 allele is sufficient to protect against ferroptotic IEC death, but insufficient to prevent PUFA-induced cytokine production. In this context, studies in heterozygous Gpx4 knockout mice did not report cell death (or related organ dysfunction $)^{11,29,48}$. Future work may define a role for ferroptotic cell death in mucosal inflammation.

Collectively, our data support a model in which dietary PUFAs elicit neutrophilic inflammation, which emanates from IECs with reduced GPX4 activity (Supplementary Fig. 12). Our findings turn the spotlight onto GPX4-restricted oxidative processes, which determine the competence of the epithelium to cope with environmental cues that inevitably occur on mucosal surfaces. Impaired GPX4 activity observed in small intestinal CD may arise from dietary long-chain fatty acids (Supplementary Fig. 5), (yet unidentified) immune mediators, bile salts ${ }^{49}$ or microbial 
Fig. 5 AA and ferric maltol induce neutrophilic inflammation in $\mathbf{G p x 4 ^ { + / - I E C }}$ mice. a Representative confocal microscopy images of 4-HNE-labeled organoids (green) from indicated genotypes after stimulation with AA and ferric iron or vehicle for $24 \mathrm{~h}$. Scale bars indicate $20 \mu \mathrm{m}$ ( $n=3$ biologically independent samples). $\mathbf{b}$ Cxcl1 expression after $48 \mathrm{~h} \mathrm{AA}$ and ferric iron stimulation of indicated organoids determined by qPCR ( $\mathrm{n}=11$ biologically independent samples). Data are presented as boxblot with median and interquartile range (25th and 75th). The whiskers represent minimal and maximal values. ${ }^{\star} P=$ 0.0303. c Model of arachidonic acid and ferric maltol gavage. d, e Representative H\&E images (d) and histology score (e) of indicated genotypes orally exposed to ferric maltol and/or AA as indicated in (c). The red arrows denote neutrophils. Scale bars indicate $100 \mu \mathrm{m}$ and $50 \mu \mathrm{m}$, respectively. Each dot represents one experimental animal ( $n=8$ mice for WT AA, $n=7$ mice for Gpx4+/-IEC AA, $n=7$ mice for WT FM, $n=7$ mice for Gpx4 $+/-I E C$ FM and $n=18$ mice for WT AA+FM and $n=25$ mice for Gpx4 $\left.{ }^{+/-I E C} \mathrm{AA}+\mathrm{FM}\right)$. ${ }^{\star \star \star} P<0.001$. f Neutrophilic infiltration of GR1 ${ }^{+}$neutrophils by flow cytometry of indicated genotypes from the experiment shown in (c). Each dot represents one experimental animal ( $n=6$ WT mice and $n=5 \mathrm{Gpx} 4^{+/-I E C}$ mice). ${ }^{\star} P=0.0228$. g Representative images of 4-HNE immunoreactivity (brown), indicative for LPO ( $n=5$ mice per group). Scale bars indicate $100 \mu m$. $\mathbf{h}$ Quantification of $C x c / 1$ expression determined by $\mathrm{QPCR}$ in intestinal epithelial scrapings of indicated genotypes from the experiment shown in (c). Each dot represents one experimental animal ( $n=10$ mice per group). $P=0.0435$. Mann-Whitney test. $\mathbf{i}$ Histology score of AA- and ferric maltol-exposed mice with or without $\alpha$ tocopherol supplementation $[0.4 \mathrm{mg} / \mathrm{ml}]$ in drinking water over the course of the experiment. Each dot represents one experimental animal $(n=4$ mice for WT + vehicle, $n=7$ mice for WT $+\alpha$-toco, $n=9$ mice for Gpx $4^{+/-I E C}+$ vehicle and $n=8$ mice for Gpx4 $4^{+/-I E C}+\alpha^{-}$toco). $P=0.0302$. j Enteritis histology score of WT and Gpx4+/-IEC mice exposed to a PUFA-enriched WD (PUFA WD) for 3 months with and without $\alpha$-tocopherol supplementation [0.4 mg/ml] in drinking water over the course of the experiment. Each dot represents one experimental animal. Median shown $(n=9$ mice for WT PUFA WD + vehicle, $n=14$ mice for Gpx4 $4^{+/-I E C}$ PUFA WD + vehicle, $n=9$ mice PUFA WD $+\alpha$-toco). ${ }^{\star *} P<0.01$. $\mathbf{k}$ Enteritis histology score of WT and Gpx4 ${ }^{+/-I E C}$ mice exposed to a PUFA-enriched WD (PUFA WD) for 3 months with and without liproxstatin-1 treatment intraperitoneally from 6 weeks [10 mg/kg] until the closure of the experiment. Each dot represents one experimental animal. Median shown ( $n=5$ mice for WT PUFA WD + vehicle, $n=8$ mice for Gpx4+/-IEC PUFA WD + vehicle, $n=9$ mice PUFA WD + liproxstatin-1). ${ }^{\star \star \star} P<0.001$. For panel $(\mathbf{e}),(\mathbf{f}),(\mathbf{h})$, and (i) data are presented as mean \pm SEM. For panel $(\mathbf{b}),(\mathbf{f})$ and $(\mathbf{h})$ unpaired two-tailed Student's $t$ test and for panel (e) and (i-k) one-way ANOVA with Bonferroni's multiple comparison test was used. Source data are provided as a Source Data file.

metabolites $^{50}$ that are absorbed in the small intestine. As we specifically observed impaired GPX4 activity in IECs from the inflamed (but not uninflamed) mucosa of CD patients, it appears plausible that GPX4 deficiency is no primary defect in CD but occurs secondary to insults (likely caused by yet unidentified cellular stressors) in the inflamed mucosa. We speculate that IBDrelated genetic cues may impinge on GPX4 activity during inflammation ${ }^{51}$, and that genetic and environmental mucosal insults (other than PUFAs) trigger an inflammatory response in GPX4-deficient hosts ${ }^{52}$. Future studies will advocate a critical role of oxidative processes on cellular membranes of IECs and decipher the impact of oxidized lipid species in mucosal inflammation and $\mathrm{IBD}^{53-55}$.

\begin{abstract}
Methods
Human studies. IBD patients were recruited in the gastroenterology outpatient clinic of the Medical University of Innsbruck and included when a definitive IBD diagnosis (by clinical, endoscopic, and histopathological means) was established and informed consent was obtained. Healthy controls undergoing screening colonoscopy - which lacked endoscopic and histological signs of intestinal disease -were included after informed consent was obtained. Patients were excluded if any of these criteria were not fulfilled or if the histology report and the endoscopic report conflicted. Ileal and colonic biopsies were collected from IBD patients and healthy controls, stored on ice in RPMI medium or formalin and processed the same day. One biopsy was used for mRNA isolation and one biopsy was formalinfixed and paraffin-embedded for histology. Remaining biopsies were used to isolate IECs according to the section IEC isolation. IEC suspensions were snap-frozen in lysis buffer and stored at $-80^{\circ} \mathrm{C}$ until GPX4 enzymatic activity testing (see section GPX4 enzymatic activity assay) or protein analysis by western blot (see section Immunoblot).
\end{abstract}

Mice. C57BL/6J Gpx4flox/flox mice ${ }^{27}$ were crossed with C57BL/6J Villin-Cre ${ }^{+}$mice $^{28}$

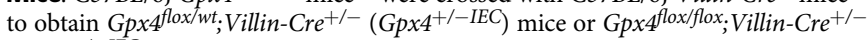
$\left(G p x 4^{-/-I E C}\right)$ mice. All experiments were performed with male and female 7 - to 10week-old mice. Littermates of the same sex were randomly assigned to experimental groups and were fed a chow diet or a Western diet (ssniff, TD88137) with or without $10 \%$ fish oil (ssniff supplementation, see Table S2). Gpx $4^{-/-I E C}$ breeding pairs were fed an $\alpha$-tocopherol enriched diet (sniff, E157892-14, $+500 \mathrm{mg} / \mathrm{kg}$ Tocopheryl Acetate) until weaning in the animal facility in Erlangen. In all other experiments $G p x 4^{+/-I E C}$ mice and WT controls were co-housed under SPF conditions (ZVTA) at the Medical University of Innsbruck.

Mouse treatment. 7- to 10-week-old WT and Gpx $4^{+/-I E C}$ mice received daily ferric maltol (0.4 mg, Shield Therapeutics PLC) and/or arachidonic acid (10 mg, Sigma, $10931)$ via oral gavage over the course of 6 days with or without a-tocopherol supplementation $(0.4 \mathrm{mg} / \mathrm{ml}$, Sigma $)$ in drinking water ad libitum. During the experiment, mice were fed a chow diet ad libitum. On day 6 mice were sacrificed for tissue collection.

Dextran sodium sulfate (DSS) colitis was induced in 8-week-old co-housed WT and Gp $x 4^{+/-I E C}$ mice using 3\% DSS (MP Biomedicals, 02160110) given in drinking water ad libitum for five consecutive days followed by tap water for the rest of the experiment. Disease activity was assessed as previously described ${ }^{56}$.

To investigate the influence of a Western diet, 7- to 10-week-old WT and Gp $x 4^{+/-I E C}$ mice were fed a Western diet (ssniff, TD88137) with or without $10 \%$ fish oil (ssniff supplementation, see Table S2) and compared to mice receiving a low-fat diet (ssniff, CD88137) for 3 months ad libitum with or without $\alpha$ tocopherol supplementation $(0.4 \mathrm{mg} / \mathrm{ml}$, Sigma $)$ in drinking water ad libitum. Bodyweight was assessed weekly. After 3 months mice were sacrificed for tissue collection.

To test the anti-inflammatory effect of Liproxstatin-1 7- to 10-week-old WT and Gpx $4^{+/-I E C}$ mice were fed a Western diet (ssniff, TD88137) with $10 \%$ fish oil (ssniff supplementation, see Table S2) for 3 months ad libitum. After 6 weeks of feeding, mice received $10 \mathrm{mg} / \mathrm{kg}$ Liproxstatin-1 (Cayman Chemicals, 17730) or vehicle (5\% DMSO in PBS) every other day by i.p. injection until closure of the experiment.

Blood count. Heparin blood samples were used to perform complete blood count analysis on a Vet-ABC animal blood counter (scil animal care company GmbH, Viernheim, Germany).

Cell culture. MODE-K cells (Mus musculus, small IECs, kindly provided by D. Kaiserlian) were cultured in high-glucose DMEM (Lonza, BE12-604F), 10\% FCS (Biochrome, S0115), HEPES (10 mM, Biochrome, L1613), non-essential amino acids $(1 \mathrm{mM}$, Gibco, 11140-035), 100U/ml penicillin and $100 \mu \mathrm{g} / \mathrm{ml}$ streptomycin (1\% Biochrome, 0257 F). HEK293T cells (Homo sapiens, female, embryonic kidney cells, ATCC, CRL-1573) were cultured in high-glucose DMEM (Lonza, BE12604F), 10\% FCS (Biochrome, S0115), HEPES (10 mM, Biochrome, L1613), nonessential amino acids ( $1 \mathrm{mM}$, Gibco, 11140-035), 100U/ml penicillin and $100 \mu \mathrm{g} / \mathrm{ml}$ streptomycin ( $1 \%$ Biochrome, $0257 \mathrm{~F}$ ) and $2 \mathrm{mM}$ sodium pyruvate ( $1 \%$ Biochrome, $\mathrm{L} 0473$ ). Cells were cultured at $37^{\circ} \mathrm{C}$ in $5 \% \mathrm{CO}_{2}$.

Reagents. The following reagents were used for cell stimulation: deferoxamine (0.1$20 \mu \mathrm{M}$, Sigma, D9533), B-thujaplicin (Hinokitol, Sigma, 469521), deferasirox (Exjade $^{\circledast}$, Novartis), fe(III) sulfate hydrate $(2-20 \mu \mathrm{M}$, Sigma, F0638), arachidonic acid (AA, $20 \mu \mathrm{M}$, Sigma, A3611), docosahexaenoic acid (DHA, $500 \mu \mathrm{M}$, Sigma, D2534), palmitic acid (PA, $250 \mu \mathrm{M}$, Sigma, P5585), oleic acid (OA, $250 \mu \mathrm{M}$, Sigma, O1008), palmitoleic acid (POA, $100 \mu \mathrm{M}$, Sigma P9417), stearidonic acid (SDA, $50 \mu \mathrm{M}$, Sigma SMB00291), eicosapentaenoic acid (EPA, $100 \mu \mathrm{M}$. Sigma E2011), docosapentaenoic acid (DPA, $25 \mu \mathrm{M}$, Sigma D1797) ferrostatin-1 (fer-1, 0.01-1 $\mu \mathrm{M}$, Sigma, SML0583), a-tocopherol (a-toco, 0.1-1 $\mu \mathrm{M}$, Sigma, T3251), Z-VAD-FMK $(0.1-100 \mu \mathrm{M}, \mathrm{BD}$ Bioscience, 550337), TNFa (50 ng/ml, PeproTech), IFN $\gamma(50 \mathrm{ng} / \mathrm{ml}, \mathrm{R} \& D)$, IL-1 $\beta$ (10 ng/ml, PeproTech), LPS (100 ng/ml, Sigma L4524), IFN $\beta$ (2500U/ml, PBL, 12401-1), IL-4 (10 ng/ml, PeproTech 214-14), IL-6 (20 ng/ml, PeproTech 216-16), 
a

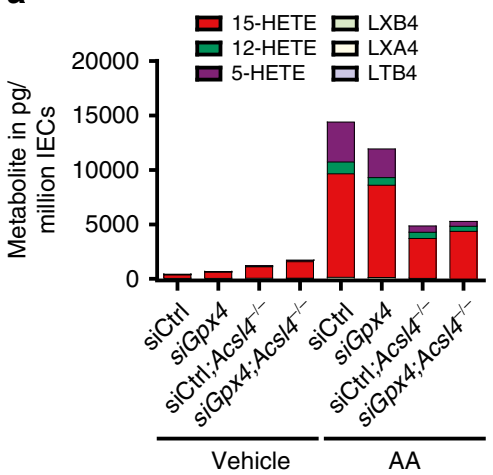

b

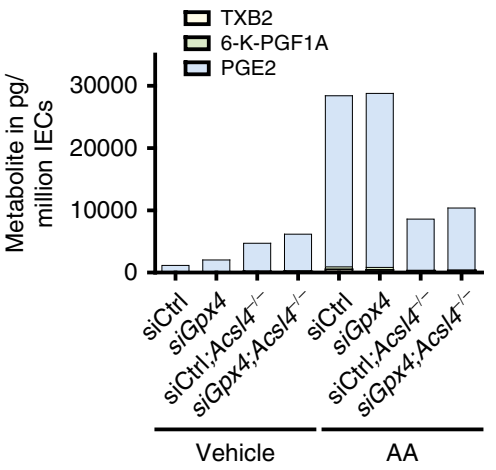

C

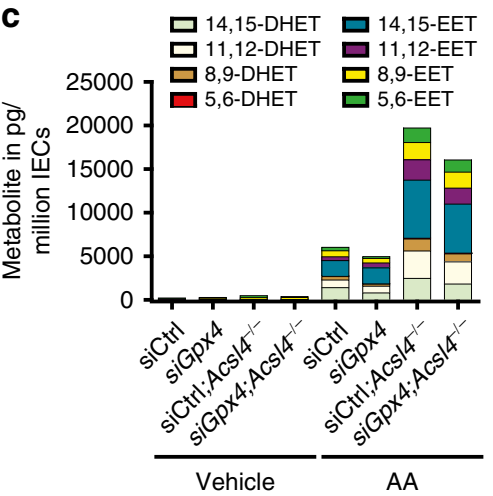

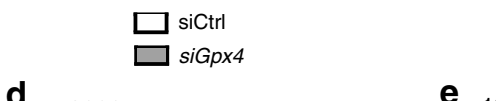

d

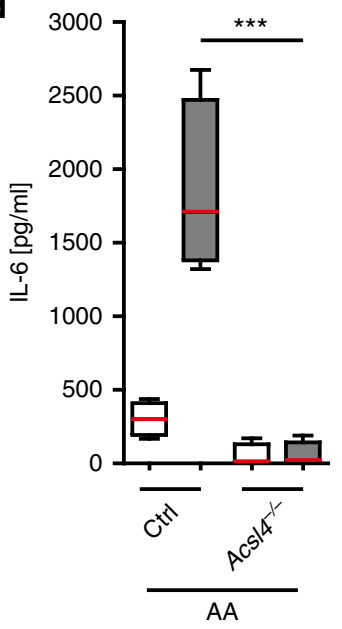

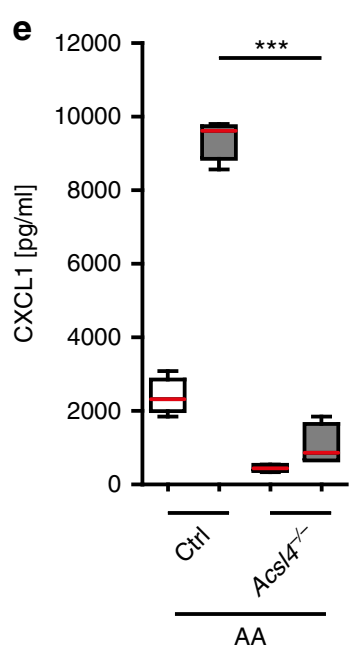

$f$

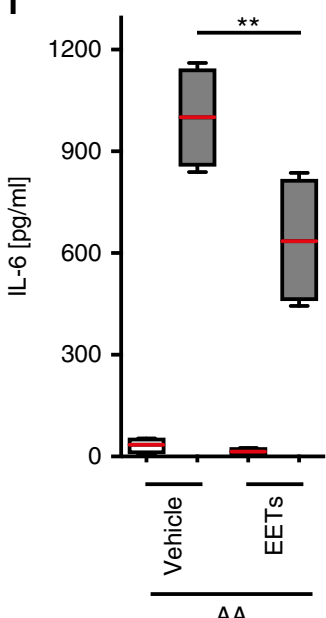

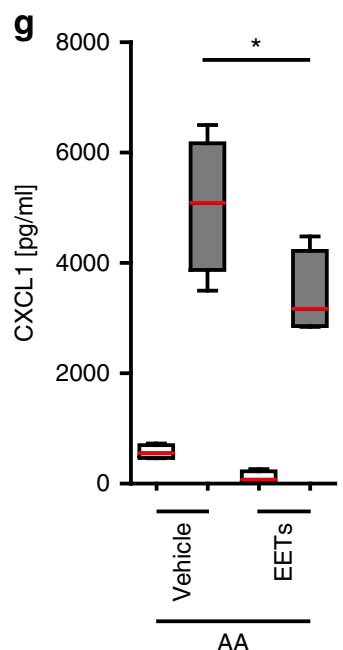

Fig. 6 ACSL4 governs AA-induced cytokine production partly by modulation of AA metabolism. a-c Quantification of LOX (a), COX (b), and P450 (c) AA metabolites in siGpx4 and siCtrl IECs with or without deletion of Acs $/ 4$ and AA stimulation for $24 \mathrm{~h}$. Three independent experiments were performed and pooled for metabolite analysis by LC-MS/MS. d, e Quantification of IL-6 and CXCL1 in the supernatant of siGpx4 and siCtrl IECs with or without deletion of Acs $/ 4$ and AA stimulation for $24 \mathrm{~h}$ ( $n=4$ biologically independent experiments). ${ }^{\star \star \star} P<0.001$. $\mathbf{f}, \mathbf{g}$ Quantification of IL-6 and CXCL1 in siGpx4 and siCtrl IECs after $24 \mathrm{~h} \mathrm{AA}$ stimulation co-treated with a combination of EETs (0.25 $\mu \mathrm{M}$ of (+/-) 8(9)-EET, (+/-) 11(12)-EET and (+/-) 14(15)-EET) ( $n=4$ biologically independent experiments). ${ }^{\star \star} P=0.0011$ and ${ }^{\star} P=0.0242$. For panel $(\mathbf{d}-\mathbf{g})$ data are presented as boxblot with median and interquartile range (25th and 75th). The whiskers represent minimal and maximal values. One-way ANOVA with Bonferroni's multiple comparison test. Source data are provided as a Source Data file.

IL-22 (10 ng/ml, PeproTech 210-22), cholic acid (1 $\mu$ M, Sigma C9282), deoxycholic acid $(1 \mu \mathrm{M}$, Sigma D2510), ursodeoxycholic acid $(1 \mu \mathrm{M}$, Sigma U5127), $(+/-) 8(9)$ and $(+/-) 11(12)$ - and $(+/-) 14(15)$-EETs $(0.25 \mu \mathrm{M}$ Cayman Chemicals, 50511,

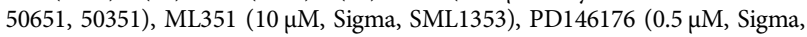
P4620), zileuton (10 $\mu$ M, Sigma, Z4277), NCTT-956 (2 $\mu$ M, Sigma, SML0499), piroxicam (20 $\mu \mathrm{M}$, Sigma, P5654), 5-HETE (200nM-1 $\mu$ M, Cayman Chemicals, 34210), 15-HPETE (200nM-1 $\mu \mathrm{M}$, Cayman Chemicals, 44720), PGE2 (50 ng/ml, Sigma,

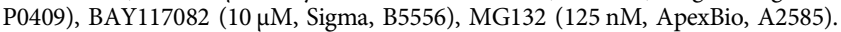

siRNA silencing. MODE-K IECs were seeded on 6-well plates at $~ 70 \%$ confluence for siRNA silencing with either Gpx4 siRNA (siGpx4, Ambion, s122098), Alox12 (siAlox12, Ambion, s62261), Alox15 (siAlox15, Ambion, s62271) or scrambled control siRNA (siCtrl, Ambion 4390843) and RNAiMAX (Thermo Fisher Scientific, 13778100) transfection over $48 \mathrm{~h}$ unless otherwise indicated, according to the recommended protocol.

CRISPR Cas9 gene editing. Target genes were disrupted in MODE-K IECs using the CRISPR/Cas9 system ${ }^{26}$. For each gene three guide RNAs (gRNA) targeting different exons of the target gene were designed. gRNAs with specific overhangs were annealed into a BsmBI-digested plentiCRISPRv2 plasmid (Addgene \#52961, gift from Feng Zhang) ${ }^{57}$. Vectors were transfected into HEK293T cells (ATCC ${ }^{\circledR}$ CRL-1573 ${ }^{\text {I }}$ ) to produce viral particles. Harvested supernatants were used for gene knockout. For transfection, MODE-K cells were seeded on 6-well plates and infected with viral particles containing the constructed vectors. The guide RNA sequence used for studies in Gpx4-/- IECs was CGTGTGCATCGTCACCAACG, which targeted exon 1. The guide RNA sequence used for studies in Acsl4-/- IECs was CAATAGAGCAGAGTACCCTG, which targeted exon 6. To generate Gp $x 4^{-1-}$ and $\mathrm{Acsl}^{-/-}$clones by CRISPR Cas9 gene editing, transfection was performed for $48 \mathrm{~h}$ followed by puromycin (Gibco, A1113803) selection for 10 days and subsequent seeding onto 96-well plates with one cell per well for expansion.

Human intestinal epithelial organoids. Human organoids were cultured from IEC isolates of biopsy specimens retrieved from endoscopy of CD patients and healthy controls using IntestiCult Organoid Growth Medium (Stemcell Technologies) and a protocol adapted from the manufacturer's instructions. Briefly, biopsies were flushed with $10 \mathrm{ml}$ of ice cold PBS and minced into the small pieces. Tissue was then transferred to $5 \mathrm{ml}$ Gentle Cell Dissociation Reagent (Stemcell Technologies) and incubated at $4{ }^{\circ} \mathrm{C}$ on a rocking platform for $30 \mathrm{~min}$. After centrifugation at $4{ }^{\circ} \mathrm{C}$ and $290 \mathrm{~g}$ for $5 \mathrm{~min}$ supernatant was removed and crypts were transferred to $1 \mathrm{ml}$ of ice cold 1\% BSA/DMEM. Crypts were dissolved by gentle mixing and passed through a $70 \mu \mathrm{m}$ cell strainer. Seeding was identical to that for mouse organoids (see below). Human organoids were passaged with a split ratio of $1: 3$ every $7-14$ days.

Mouse intestinal epithelial organoids. Intestinal epithelial organoids were cul-

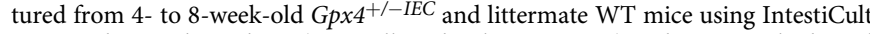
Organoid Growth Medium (Stemcell Technologies, 06005) and a protocol adapted from manufacturer's instructions as initially described ${ }^{58}$. Briefly, small intestines were flushed with ice cold PBS, minced to pieces of approximately $2-3 \mathrm{~mm}$ in size and washed up to five times with $10 \mathrm{ml}$ ice cold PBS. Samples were transferred to 
$2 \mathrm{mM} \mathrm{EDTA} / \mathrm{PBS}$ and incubated at $4{ }^{\circ} \mathrm{C}$ on a rocking platform for $30 \mathrm{~min}$. After sedimentation supernatant was removed and crypts eluted in $10 \mathrm{ml}$ PBS by shaking vigorously and passing crypts through a $70 \mu \mathrm{m}$ cell strainer to obtain Fraction 1. This process was repeated three times to obtain Fractions 2-4. Fractions were analyzed under a light microscope and the optimal fraction was chosen to obtain crypts for organoid culture by centrifugation at $290 \mathrm{~g}$ for $5 \mathrm{~min}$ at $4{ }^{\circ} \mathrm{C}$. Crypts $(N=500)$ per well were seeded in $50 \mu$ Matrigel $(\mathrm{BD}, 356231)$ on a prewarmed 24-well plate and allowed to solidify for $10 \mathrm{~min}$ at $37^{\circ} \mathrm{C}$, after which $500 \mu \mathrm{l}$ IntestiCult Growth Medium supplemented with $100 \mathrm{U} / \mathrm{ml}$ penicillin and $100 \mu \mathrm{g} / \mathrm{ml}$ streptomycin (Biochrome, 0257F) was added. Medium was exchanged three times per week and organoids passaged with a split ratio of 1:6 every 7-14 days (Supplementary Fig. 8A-G).

Stimulation of mouse organoids. Before stimulation, organoids were allowed to establish for 6 days. Organoids were stimulated with arachidonic acid (AA, $100 \mu \mathrm{M}$, Sigma, A3611) and Fe(III) sulfate (5 $\mu \mathrm{M}$, Sigma, F0638) or vehicle for indicated time periods depending on the experiment setting. Medium was replaced every $24 \mathrm{~h}$. For RNA extraction TRIzol reagent (Invitrogen, 15596026) was used according to the manufacturer's instructions.

Immunofluorescence Imaging. Organoids were cultured with $15 \mu$ l Matrigel (BD, 356231) on chamber slides (Falcon, 354108) covered with $200 \mu$ IntestiCult Growth Medium supplemented with $100 \mathrm{U} / \mathrm{ml}$ penicillin and $100 \mu \mathrm{g} / \mathrm{ml}$ streptomycin (Biochrome, 0257 F). Medium was exchanged three times a week and organoids were allowed to establish for 6 days. Organoids were stimulated as described above for $24 \mathrm{~h}$, washed with PBS and fixed with $4 \%$ PFA at room temperature for 20 min, washed again twice with PBS and permeabilized with PBSTriton X $0.5 \%$ for $20 \mathrm{~min}$ at room temperature. Organoids were washed with IF buffer (PBS, Triton X $0.3 \%$, Tween $0.05 \%$ ), blocked with blocking solution (PBSTriton X $0.3 \%$, Tween $0.05 \%$, BSA $1 \%$ ), followed by another wash with IF buffer and primary antibody in blocking solution was added over night at $4{ }^{\circ} \mathrm{C}$. Organoids were washed three times with IF buffer and secondary antibody was added for $1 \mathrm{~h}$, washed three times with IF buffer, mounted (Invitrogen, P36962) and analyzed with a Zeiss Axio Observer Z1 confocal microscope and Zen 2012 software. Intestinal sections were cryopreserved in O.C.T., acetone-fixed, stained with antiGR-1 and analyzed with the confocal microscope stated above.

The following antibodies were used for immunofluorescence: anti-4HNE (1:200, Abcam, ab46545), anti-GPX4 (1:400, Abcam, ab125066) and anti-GR-1 (1:100, BioLegend, 108413) as primary antibodies and anti-rabbit IgG Alexa Fluor 488 (1:1000, Invitrogen, A-11034) and anti-rat IgG Alexa Fluor 594 (1:1000, Invitrogen, A-11007) as secondary antibody.

Histology. Formalin-fixed paraffin-embedded sections were hematoxylin \& eosin (H\&E)-stained and assessed for inflammation by an expert pathologist using a light microscope (Zeiss, Germany). Images were captured with a Zeiss AxioCam. In AA and FM gavage experiments, acute inflammation was quantified by determining the number of neutrophilic spots ( $>3$ neutrophils in one visual field on $\times 20$ magnification) per section. We did not note mononuclear infiltration, hyperplasia or ulceration in our AA and FM exposure experiments. DSS colitis severity and enteritis evaluation was performed as reported ${ }^{59}$ : Briefly, a semi-quantitative scoring system was used that was composed of five histological subscores. Histological subscores ( 0 , absent; 1 , mild; 2 , moderate; 3 , severe) were mononuclear cell infiltration, crypt hyperplasia, epithelial injury/erosion, polymorphonuclear cell infiltration and transmural inflammation. The sum of these subscores was multiplied by a factor that reflected the extent of inflammation along the intestine $(1$, $10 \% ; 2,10-25 \% ; 3,25-50 \%$; and $4,>50 \%$ ).

Immunohistochemistry, TUNEL, BrdU, and PAS labeling. Formalin-fixed paraffin-embedded sections were deparaffinised, rehydrated, antigen-retrieved for $15 \mathrm{~min}$ in sodium citrate at subboiling temperatures and peroxidase-blocked. The primary antibody was incubated over night at $4{ }^{\circ} \mathrm{C}$ and the secondary biotinylated antibody mediated horseradish peroxidase (HRP)-driven 3,3'-diaminobenzidine (DAB, DAKO, K3468) turnover, which resulted $\mathrm{n}$ brown labeling of immunoreactive cells. Stained sections were analyzed with a light microscope (Zeiss, Germany) and captured with a Zeiss AxioCam. TUNEL labeling was performed according to the manufacturer's instructions (Roche, 11684817910). TUNELpositive cells were quantified in 50 consecutive crypts and depicted as TUNEL ${ }^{+}$ cells of total IECs. Proliferation of IECs was investigated by intraperitoneal injection of 5-bromodeoxyuridine (BrdU, BD Pharmigen, 550891) and detected after $24 \mathrm{~h}$ using a BrdU in situ detection kit (BD Pharmigen, 550803). BrdU ${ }^{+}$cells of total IEC along the villus-crypt axis were analyzed. PAS reaction was performed according to a standard protocol and $\mathrm{PAS}^{+}$cells were counted in $50 \mathrm{crypt} /$ villus axes.

The following antibodies were used for immunohistochemistry: anti-GPX4 (1:400, Abcam, ab125066), anti-4HNE (1:400, Abcam, ab46545) and anti-MPO (1:200, Dako, IS511) were employed with a secondary biotinylated antibody (Vector, MP-7401).
Mouse IEC isolation. Mouse IEC isolation protocol was performed as previously described $^{59}$. Briefly, PBS-flushed and longitudinally cut intestinal pieces were vortexed in ice cold PBS for $5 \mathrm{~min}$, transferred to $30 \mathrm{mM}$ EDTA, and vortexed for $5 \mathrm{~min}$. The supernatant was collected and the procedure was repeated for a total of four times. Supernatants were microscopically checked for crypt IEC enrichment and then spun down at $800 \mathrm{~g}$ for protein extraction or flow cytometry labeling.

Human IEC isolation. The biopsies were moved from RPMI (Biochrome, FG1385) to HBSS-CMF buffer (Gibco, 14175-053, 0.5\% BSA, 2 mM EDTA and DTT). Samples were incubated on a shaker for $20 \mathrm{~min}$ at room temperature. The samples were then vortexed vigorously and supernatant (containing IECs) was collected through a $100 \mu \mathrm{m}$ cell strainer, which was repeated for a total of three times. Supernatant was then spun down at $300 \mathrm{~g}$ and the pelle $\mathrm{t}$ was used for GPX4 activity assay and western blot analysis as detailed in the respective sections.

Murine LPMC isolation. Mouse LPMCs were isolated according to previously published protocols ${ }^{60,61}$. In short, the proximal small intestine was flushed with ice cold PBS, opened longitudinally, cut into small pieces and transferred to HBSS (Gibco, 14175-053) containing 10\% FCS, DTT $(1 \mathrm{mM})$ and EDTA $(2 \mathrm{mM})$ followed by shaking for $20 \mathrm{~min}$ at room temperature. Samples were vortexed to remove IELs and the tissue was washed and collected in IMDM (Gibco, 21056-023) $20 \%$ FCS. Tissue was washed and $10 \mathrm{U} / \mathrm{ml}$ DNAse $(10 \mathrm{U} / \mathrm{ml}$, Sigma, D8764) and $128 \mathrm{U} / \mathrm{ml}$ collagenase $(128 \mathrm{U} / \mathrm{ml}$, Sigma, C1889) digested on a shaker for $60 \mathrm{~min}$ at $37^{\circ} \mathrm{C}$. Cells were passed through cell strainers $(100 \mu \mathrm{m})$ and washed twice before transferring to cytometry buffer for staining (see below).

LPO and cell death labeling. Cells derived from cell culture or from IEC isolation procedures were incubated with BODIPY 581/591 C11 or the surface labeling antibodies (see below) at $37^{\circ} \mathrm{C}$ in the dark for ten to $30 \mathrm{~min}$ in flow cytometry buffer (2\% FCS, 2 mM EDTA in PBS). Cells were subsequently washed with PBS, resuspended in FACS buffer and transferred through a $40 \mu \mathrm{m}$ cell strainer for flow cytometry. Annexin V, PI or 7AAD were used for cell death analysis.

Flow cytometry analysis. For LPO analysis, BODIPY-positive cells among DAPInegative cells were analyzed as compared to a control sample using BODIPY measurement. For cell death analysis, debris was excluded using FSC/SSC characteristics; Annexin V and PI or 7AAD positivity was determined by flow cytometry.

FACS gating strategy. The gating strategy for analyzing the mucosal cellular infiltrate is depicted in Supplementary Fig. 9A, E. Briefly, cells were gated using FSC/SSC characteristics. Singlets were selected by comparing FSC width and FSC area. Neutrophils were identified as $\mathrm{CD}_{4} 5^{+}, \mathrm{Lin1}^{-}(\mathrm{Lin} 1=\mathrm{CD} 3, \mathrm{CD} 19, \mathrm{CD} 49 \mathrm{~b}$, DAPI) and $\mathrm{GR}^{+}$cells. Macrophages were identified by $\mathrm{CD}_{4} 5^{+}, \mathrm{Lin}^{-}, \mathrm{GR}^{-}$, $\mathrm{CD}_{11 \mathrm{~b}^{+}, \mathrm{MerTK}^{+} \text {. Monocytes were characterized by CD45 }}+$, Lin1 ${ }^{-}, \mathrm{GR}^{-}$, Ly6C ${ }^{\text {hi }}$. Dendritic cells were characterized by $\mathrm{CD} 45^{+}, \mathrm{Lin1}^{-}, \mathrm{GR} 1^{-}, \mathrm{CD} 11 \mathrm{c}^{+}$and $\mathrm{MHCII}^{+}$. T helper cells were identified by Lin2 ${ }^{-}(\mathrm{Lin} 2=\mathrm{CD} 11 \mathrm{c}, \mathrm{F} 4 / 80$, GR1, DAPI), $\mathrm{CD}^{+}, \mathrm{CD}^{-} 9^{-}, \mathrm{CD}^{+}$. Cytotoxic $\mathrm{T}$ cells were identified by $\mathrm{Lin}^{-}{ }^{-}, \mathrm{CD}^{+}$, $\mathrm{CD}^{-} 9^{-}, \mathrm{CD}^{+}$. B cells were defined as $\mathrm{Lin2}^{-}, \mathrm{CD}^{-}, \mathrm{CD} 19^{+}$. Details of antibodies used are found in Supplementary Table 4.

RNA extraction and qRT-PCR. RNA was isolated from 6-well plates, epithelial scrapings or human intestinal biopsies using an RNeasy mini kit (Quiagen 74104). RNA was transcribed into cDNA using M-MLV reverse transcriptase (Invitrogen 28025013). Quantitative real time PCR was performed with SYBR green mastermix (Eurogentec RT-SY2X-06+WOULR) on a MX3005 Stratagene cycler (Agilent). The primer used in this study can be found in Supplementary Table 4.

Immunoblot. Western blot analysis was performed according to standard protocols (Bio-Rad Laboratories). Briefly, isolated cells (from culture plates or scrapings) were lysed in RIPA buffer (50 mM Tris, pH 7.4, $150 \mathrm{mM} \mathrm{NaCl}, 1 \%$ Nonidet P-40, $0.5 \%$ sodium deoxycholate, and $0.1 \%$ SDS) or M-Per (Thermo Fisher Scientific, 78501) and supplemented with protease and phosphatase inhibitors (Thermo Fisher Scientific, 78443). Protein quantity was determined by Bradford assay (BioRad Laboratories, 5000006) and equal amounts of protein were denatured at $95^{\circ} \mathrm{C}$ in Laemmli buffer, resolved on SDS-PAGE and transferred to a polyvinylidene fluoride membrane (Sigma, GE10600023). After blocking the membrane in 5\% skim milk, primary antibody was incubated over night at $4{ }^{\circ} \mathrm{C}$. Signal was visualized with HRP-conjugated secondary antibodies (Cell Signalling Technology, 7074) and ECL Select Western Blotting Detection Reagent (Amersham, RPN2235). Densitometry of immunoblots was performed with ImageJ. All uncropped and unedited blots are available in the Source Data. The following antibodies were used: antiGPX4 (1:2000, Abcam, ab125066), anti-ACSL4 (1:1000, Abcam, ab155282), antiTfR (1:1000, Invitrogen, H68.4), anti-FPN1 (1:1000, Eurogentec, Liege, Belgium) anti-ferritin (1:1000, Sigma, F5012), anti-phospho-NF-kB p65 (1:1000, Cell Signalling Technology, 3039), anti-NF-kB p65 (1:1000, Cell Signalling Technology, 8242), with anti-GAPDH (1:2000, Cell Signalling Technology, 2118) or anti-ß3-actin (1:2000, Sigma, A2066 and Abcam, ab49900) as loading control. 
Cytokine quantification. Cellular supernat ants were collected, centrifuged at $300 \mathrm{~g}$ for $5 \mathrm{~min}$ and stored at $-20^{\circ} \mathrm{C}$. Cytokine quantification was performed by ELISA (IL-6, BD Biosciences, 555240; CXCL1/KC, R\&D, DY453) according to the manufacturer's protocol.

Cell viability assay. Cells were seeded on 96 -well plates (2000 cells per well) and treated with siRNA for $48 \mathrm{~h}$. Cell viability was assessed by AlamarBlue turnover (Thermo Fisher Scientific, DAL1025) according to the manufacturer's recommendations.

Quantification of iron uptake and release. Iron uptake and release were performed as previously described ${ }^{62}$. Briefly, MODE-K IECs were silenced for $48 \mathrm{~h}$ with siGpx4 or siCtrl and washed with high-glucose DMEM (1\% FCS, $1 \%$ penicillin/streptomycin, $25 \mathrm{mM}$ HEPES). To determine non-transferrin-bound iron uptake and release, cells were incubated with $5 \mu \mathrm{M}^{59}$ ferric chloride (Perkin Elmer, NEZ037) for $2 \mathrm{~h}$. After washing, cells were transferred to high-glucose DMEM (1\% FCS, $1 \%$ penicillin/streptomycin, $25 \mathrm{mM}$ HEPES) and incubated for $1 \mathrm{~h}$. Iron uptake and release were measured with a $\gamma$ counter (Perkin Elmer). Uptake and release in CPM (counts per minute) were normalized to protein quantity as determined by BCA Assay (Thermo Fisher Scientific, 23225).

GPX4 enzymatic activity assay. Cells were collected in lysis buffer (100 mM Tris pH 7.6, $5 \mathrm{mM}$ EDTA, $1 \mathrm{mM} \mathrm{NaN}_{3}$ and $0.1 \%$ peroxide-free Triton-X100). Lysates were complemented with $0.6 \mathrm{U} / \mathrm{mL}$ glutathione reductase (Sigma, G3664), $0.2 \mathrm{mM}$ nicotinamide adenine dinucleotide phosphate hydrogen (NADPH, Sigma, N7505), $3 \mathrm{mM}$ reduced glutathione (GSH, Sigma, G4251) and $200 \mu \mathrm{M}$ of the substrate cumene hydroperoxide (CHP, Sigma, 247502). NADPH turnover was measured on an Infinite 200PRO reader (Tecan) at $340 \mathrm{~nm}$ over $10 \mathrm{~min}$ at $37^{\circ} \mathrm{C}$. Enzymatic activity was calculated after subtracting absorbance decay obtained from buffer without cell lysates by using NADPH extinction coefficient of $6220 / \mathrm{M} / \mathrm{cm}$ and by normalizing to total protein content ${ }^{63}$.

LC-MS/MS analysis. The extraction protocol and analysis of bioactive lipids by LC-MS/MS were performed by Ambiotis SAS (Toulouse, France). Briefly cell pellets were supplemented with methanol and spiked with deuterated internal standards (ISTD) (PGE2-d4, 5-HETE-d8, LtB4-d4, LXA4-d5). After standing at $-20^{\circ} \mathrm{C}$ for $1 \mathrm{~h}$ and centrifugation, supernatants were diluted with $\mathrm{H} 2 \mathrm{O}$ and subjected to solid phase extraction (SPE) on an HLB 96 well plate (Oasis). Methyl formate-eluted lipid mediators were concentrated by a nitrogen stream evaporator before LC-MS/MS analysis. LC-MS/MS experiment was performed on a 1290 Infinity U-HPLC system (Agilent Technologies, Santa Clara, CA, USA) mounted with a Kinetex Biphenyl column $(2.1 \mathrm{~mm}, 50 \mathrm{~mm}, 1.7 \mu \mathrm{m}$, Phenomenex), maintained at $50{ }^{\circ} \mathrm{C}$. The U-HPLC system was coupled to a 6490 triple quadrupole MS (Agilent Technologies), equipped with electrospray ionization source, performed in negative ion mode. Analyses were performed in multiple reaction monitoring detection mode by use of nitrogen as collision gas. Peak detection, integration, and quantitative analysis were done by use of MassHunter Quantitative Analysis Software (Agilent Technologies). Results are expressed in a quantitative manner (i.e. $\mathrm{pg} / \mathrm{sample}$ ). Validation and extraction efficacy of the extraction protocol was validated in Le Faouder et al ${ }^{64}$. Data was deposited in Mendeley (https://data. mendeley.com, 10.17632/k9ync2kd3g.2; Data for: Dietary lipids fuel GPX4restricted enteritis resembling $\mathrm{CD}$ ).

Statistics. Data are expressed as mean \pm standard error of mean (SEM) for all in vivo experiments. Data derived from in vitro experimentation is presented as box and whisker plot with median and first and third quartile (boundaries). The whiskers represent minimal and maximal values. Results shown are of at least three independent experiments with two technical duplicates, unless stated otherwise. Statistical significance was tested using an unpaired two-tailed Student's $t$ test, a Mann-Whitney U test, a one-way or two-way ANOVA with Bonferroni correction or a Kruskal-Wallis test followed by Dunn's correction as indicated in the figure legend. Significance was considered $P<0.05$. Grubbs' Test allowed determination and exclusion of one significant outlier in a parametric sample set. Graph Pad Prism version 5.04 was used for statistical analysis.

Study approval. We confirm that we comply with all relevant ethical regulations regarding the use of research animals and human study participants. Human studies were approved by the Ethics Committee of the Medical University of Innsbruck (UN4994) and informed consent was obtained prior to sample collection. Mouse experiments were performed in accordance with institutional guidelines of the Medical University of Innsbruck and following approval by federal authorities (BMWFW-66.011/0061-WF/V/3b/2016, BMBWF-66.011/0085-V/3b/2018 and BMBWF-66.011/0160-V/3b/2019 (Innsbruck); 55.2.2-2532-2-1043 (Erlangen)).

Reporting summary. Further information on research design is available in the Nature Research Reporting Summary linked to this article.

\section{Data availability}

The data of this study are available from the corresponding author upon reasonable request. The dataset generated in this study are deposited in a publicly available platform (https://data.mendeley.com, 10.17632/k9ync2kd3g.2; Data for: Dietary lipids fuel GPX4restricted enteritis resembling Crohn's disease). The source data for Figs. 1-6 and Supplementary Fig. S1-11 are provided in the Source Data File.

Received: 26 May 2019; Accepted: 23 March 2020; Published online: 14 April 2020

\section{References}

1. Stockwell, B. R. et al. Ferroptosis: a regulated cell death nexus linking metabolism, redox biology, and disease. Cell 171, 273-285 (2017).

2. Kagan, V. E. et al. Oxidized arachidonic and adrenic PEs navigate cells to ferroptosis. Nat. Chem. Biol. 13, 81-90 (2017).

3. Seiler, A. et al. Glutathione peroxidase 4 senses and translates oxidative stress into 12/15-lipoxygenase dependent- and AIF-mediated cell death. Cell Metab. 8, 237-248 (2008)

4. Magtanong, L., Ko, P. J. \& Dixon, S. J. Emerging roles for lipids in nonapoptotic cell death. Cell Death Differ. 23, 1099-1109 (2016).

5. Ursini, F., Maiorino, M. \& Gregolin, C. The selenoenzyme phospholipid hydroperoxide glutathione peroxidase. Biochim. Biophys. Acta 839, 62-70 (1985).

6. Doll, S. et al. ACSL4 dictates ferroptosis sensitivity by shaping cellular lipid composition. Nat. Chem. Biol. 13, 91-98 (2017).

7. Kang, M. J. et al. A novel arachidonate-preferring acyl-CoA synthetase is present in steroidogenic cells of the rat adrenal, ovary, and testis. Proc. Natl Acad. Sci. USA 94, 2880-2884 (1997).

8. Chen, L., Hambright, W. S., Na, R. \& Ran, Q. Ablation of the ferroptosis inhibitor glutathione peroxidase 4 in neurons results in rapid motor neuron degeneration and paralysis. J. Biol. Chem. 290, 28097-28106 (2015).

9. Sengupta, A. et al. Targeted disruption of glutathione peroxidase 4 in mouse skin epithelial cells impairs postnatal hair follicle morphogenesis that is partially rescued through inhibition of COX-2. J. Investigative Dermatol. 133, 1731-1741 (2013).

10. Linkermann, A. et al. Synchronized renal tubular cell death involves ferroptosis. Proc. Natl Acad. Sci. USA 111, 16836-16841 (2014).

11. Friedmann Angeli, J. P. et al. Inactivation of the ferroptosis regulator Gpx4 triggers acute renal failure in mice. Nat. Cell Biol. 16, 1180-1191 (2014).

12. Wenzel, S. E. et al. PEBP1 wardens ferroptosis by enabling lipoxygenase generation of lipid death signals. Cell 171, 628-641 e626 (2017).

13. Alvarez, S. W. et al. NFS1 undergoes positive selection in lung tumours and protects cells from ferroptosis. Nature 551, 639-643 (2017).

14. Hangauer, M. J. et al. Drug-tolerant persister cancer cells are vulnerable to GPX4 inhibition. Nature 551, 247-250 (2017).

15. Uhlig, H. H. \& Powrie, F. Translating immunology into therapeutic concepts for inflammatory bowel disease. Annu. Rev. Immunol. 36, 755-781 (2018).

16. $\mathrm{Chu}, \mathrm{H}$. et al. Gene-microbiota interactions contribute to the pathogenesis of inflammatory bowel disease. Science 352, 1116-1120 (2016).

17. Devkota, S. et al. Dietary-fat-induced taurocholic acid promotes pathobiont expansion and colitis in Il10-/- mice. Nature 487, 104-108 (2012).

18. Chassaing, B. et al. Dietary emulsifiers impact the mouse gut microbiota promoting colitis and metabolic syndrome. Nature 519, 92-96 (2015).

19. Patterson, E., Wall, R., Fitzgerald, G. F., Ross, R. P. \& Stanton, C. Health implications of high dietary omega-6 polyunsaturated fatty acids. J. Nutr. Metab. 2012, 539426 (2012)

20. de Silva, P. S. et al. An association between dietary arachidonic acid, measured in adipose tissue, and ulcerative colitis. Gastroenterology 139, 1912-1917 (2010).

21. Nishida, T. et al. Increased arachidonic acid composition of phospholipids in colonic mucosa from patients with active ulcerative colitis. Gut 28, 1002-1007 (1987).

22. Ungaro, F., Rubbino, F., Danese, S. \& D'Alessio, S. Actors and factors in the resolution of intestinal inflammation: lipid mediators as a new approach to therapy in inflammatory bowel diseases. Front Immunol. 8, 1331 (2017).

23. Jostins, L. et al. Host-microbe interactions have shaped the genetic architecture of inflammatory bowel disease. Nature 491, 119-124 (2012).

24. Pasparakis, M. \& Vandenabeele, P. Necroptosis and its role in inflammation. Nature 517, 311-320 (2015)

25. Cummings, R. J. et al. Different tissue phagocytes sample apoptotic cells to direct distinct homeostasis programs. Nature 539, 565-569 (2016).

26. Sander, J. D. \& Joung, J. K. CRISPR-Cas systems for editing, regulating and targeting genomes. Nat. Biotechnol. 32, 347-355 (2014).

27. Yoo, S. E. et al. Gpx4 ablation in adult mice results in a lethal phenotype accompanied by neuronal loss in brain. Free Radic. Biol. Med 52, 1820-1827 (2012). 
28. Madison, B. B. et al. Cis elements of the villin gene control expression in restricted domains of the vertical (crypt) and horizontal (duodenum, cecum) axes of the intestine. J. Biol. Chem. 277, 33275-33283 (2002).

29. Canli, O. et al. Myeloid cell-derived reactive oxygen species induce epithelial mutagenesis. Cancer Cell 32, 869-883 e865 (2017).

30. Yang, W. S. et al. Peroxidation of polyunsaturated fatty acids by lipoxygenases drives ferroptosis. Proc. Natl Acad. Sci. USA 113, E4966-E4975 (2016).

31. Gasche, C. et al. Ferric maltol is effective in correcting iron deficiency anemia in patients with inflammatory bowel disease: results from a phase- 3 clinical trial program. Inflamm. Bowel Dis. 21, 579-588 (2015).

32. Yang, Q. et al. Dietary intake of n-3 PUFAs modifies the absorption, distribution and bioavailability of fatty acids in the mouse gastrointestinal tract. Lipids Health Dis. 16, 10 (2017).

33. Muckenthaler, M. U., Rivella, S., Hentze, M. W. \& Galy, B. A red carpet for iron metabolism. Cell 168, 344-361 (2017).

34. Node, K. et al. Anti-inflammatory properties of cytochrome P450 epoxygenase-derived eicosanoids. Science (New York, NY) 285, 1276-1279 (1999).

35. Ganesan, B., Brothersen, C. \& McMahon, D. J. Fortification of foods with omega-3 polyunsaturated fatty acids. Crit. Rev. Food Sci. Nutr. 54, 98-114 (2014).

36. Simopoulos, A. P. An Increase in the Omega-6/Omega-3 fatty acid ratio increases the risk for obesity. Nutrients 8, 128 (2016).

37. Hou, J. K., Abraham, B. \& El-Serag, H. Dietary intake and risk of developing inflammatory bowel disease: a systematic review of the literature. Am. J. Gastroenterol. 106, 563-573 (2011)

38. Lev-Tzion, R., Griffiths, A. M., Leder, O. \& Turner, D. Omega 3 fatty acids (fish oil) for maintenance of remission in Crohn's disease. The Cochrane database of systematic reviews, CD006320, https://doi.org/10.1002/14651858. CD006320.pub4 (2014)

39. Hull, M. A. et al. Eicosapentaenoic acid and aspirin, alone and in combination, for the prevention of colorectal adenomas (seAFOod Polyp Prevention trial): a multicentre, randomised, double-blind, placebo-controlled, 2 × 2 factorial trial. Lancet 392, 2583-2594 (2018).

40. Gorard, D. A. et al. Initial response and subsequent course of Crohn's disease treated with elemental diet or prednisolone. Gut 34, 1198-1202 (1993).

41. Yamamoto, T., Nakahigashi, M., Umegae, S., Kitagawa, T. \& Matsumoto, K. Impact of elemental diet on mucosal inflammation in patients with active Crohn's disease: cytokine production and endoscopic and histological findings. Inflamm. Bowel Dis. 11, 580-588 (2005).

42. Kurashima, Y. \& Kiyono, H. Mucosal ecological network of epithelium and immune cells for gut homeostasis and tissue healing. Annu. Rev. Immunol. 35, 119-147 (2017).

43. Dennis, E. A. \& Norris, P. C. Eicosanoid storm in infection and inflammation. Nat. Rev. Immunol. 15, 511-523 (2015).

44. Moor, A. E. et al. Spatial reconstruction of single enterocytes uncovers broad zonation along the Intestinal Villus Axis. Cell 175, 1156-1167 e1115 (2018).

45. Belluzzi, A. et al. Effect of an enteric-coated fish-oil preparation on relapses in Crohn's disease. N. Engl. J. Med 334, 1557-1560 (1996).

46. Feagan, B. G. et al. Omega-3 free fatty acids for the maintenance of remission in Crohn disease: the EPIC Randomized Controlled Trials. Jama 299, 1690-1697 (2008).

47. Turner, D., Shah, P. S., Steinhart, A. H., Zlotkin, S. \& Griffiths, A. M. Maintenance of remission in inflammatory bowel disease using omega-3 fatty acids (fish oil): a systematic review and meta-analyses. Inflamm. bowel Dis. 17, 336-345 (2011).

48. Yant, L. J. et al. The selenoprotein GPX4 is essential for mouse development and protects from radiation and oxidative damage insults. Free Radic. Biol. Med 34, 496-502 (2003).

49. Maiorino, M., Roveri, A., Gregolin, C. \& Ursini, F. Different effects of Triton $\mathrm{X}-100$, deoxycholate, and fatty acids on the kinetics of glutathione peroxidase and phospholipid hydroperoxide glutathione peroxidase. Arch. Biochem Biophys. 251, 600-605 (1986).

50. Garreta, A. et al. Structure and interaction with phospholipids of a prokaryotic lipoxygenase from Pseudomonas aeruginosa. FASEB J. 27, 4811-4821 (2013).

51. Grootjans, J., Kaser, A., Kaufman, R. J. \& Blumberg, R. S. The unfolded protein response in immunity and inflammation. Nat. Rev. Immunol. 16, 469-484 (2016).

52. Bowie, A. G., Moynagh, P. N. \& O’Neill, L. A. Lipid peroxidation is involved in the activation of NF-kappaB by tumor necrosis factor but not interleukin-1 in the human endothelial cell line ECV304. Lack of involvement of $\mathrm{H} 2 \mathrm{O} 2$ in NF-kappaB activation by either cytokine in both primary and transformed endothelial cells. J. Biol. Chem. 272, 25941-25950 (1997).

53. Mills, E. L. et al. Succinate dehydrogenase supports metabolic repurposing of mitochondria to drive inflammatory macrophages. Cell 167, 457-470 e413 (2016).
54. Ip, W. K. E., Hoshi, N., Shouval, D. S., Snapper, S. \& Medzhitov, R. Antiinflammatory effect of IL-10 mediated by metabolic reprogramming of macrophages. Science 356, 513-519 (2017).

55. von Moltke, J. et al. Rapid induction of inflammatory lipid mediators by the inflammasome in vivo. Nature 490, 107-111 (2012)

56. Garrett, W. S. et al. Communicable ulcerative colitis induced by T-bet deficiency in the innate immune system. Cell 131, 33-45 (2007).

57. Sanjana, N. E., Shalem, O. \& Zhang, F. Improved vectors and genome-wide libraries for CRISPR screening. Nat. Methods 11, 783-784 (2014).

58. Sato, T. et al. Single Lgr5 stem cells build crypt-villus structures in vitro without a mesenchymal niche. Nature 459, 262-265 (2009).

59. Adolph, T. E. et al. Paneth cells as a site of origin for intestinal inflammation. Nature 503, 272-276 (2013).

60. Platt, A. M., Bain, C. C., Bordon, Y., Sester, D. P. \& Mowat, A. M. An independent subset of TLR expressing CCR2-dependent macrophages promotes colonic inflammation. J. Immunol. 184, 6843-6854 (2010).

61. Gerner, R. R. et al. NAD metabolism fuels human and mouse intestinal inflammation. Gut https://doi.org/10.1136/gutjnl-2017-314241 (2017).

62. Ludwiczek, S., Aigner, E., Theurl, I. \& Weiss, G. Cytokine-mediated regulation of iron transport in human monocytic cells. Blood 101, 4148-4154 (2003).

63. Brault, C. et al. Glutathione peroxidase 4 is reversibly induced by HCV to control lipid peroxidation and to increase virion infectivity. Gut 65, 144-154 (2016).

64. Le Faouder, P. et al. LC-MS/MS method for rapid and concomitant quantification of pro-inflammatory and pro-resolving polyunsaturated fatty acid metabolites. J. Chromatogr. B: Anal. Technol. Biomed. Life Sci. 932, 123-133 (2013).

\section{Acknowledgements}

The authors are grateful for the support received from the Austrian Science Fund (FWF, P 29379-B28), the Tyrolean Science Fund (TWF, 0404/1812), the Austrian Society of Gastroenterology and Hepatology (ÖGGH) and the European Crohn's and Colitis Organisation (ECCO) (to T.E.A.). We thank the Excellence Initiative (Competence Centers for Excellent Technologies-COMET) of the Austrian Research Promotion Agency FFG: Research Center of Excellence in Vascular Ageing Tyrol, VASCage (K-Project Nr. 843536) funded by BMVIT, BMWFW, Wirtschaftsagentur Wien and Standortagentur Tirol for their financial support (to H.T.). This work was further supported by the German Funding Agency (DFG) through the CRC1182 (C2 to F.S. \& P.R.), the excellence initiative EXC306 (to P.R.) and EXS2167 (to P.R.). We thank the German Funding Agency (DFG) through TRR241 (A03 to C.B.) and SFB1181 (C05 to C.B.). We appreciate the support of the Christian Doppler Research Foundation and the Austrian Federal Ministry of Science, Research and Economy and the National Foundation for Research, Technology and Development (to A.R.M and G.W.). We thank Cornelia Wiedner Stiftung and Deutsche Arbeitsgemeinschaft für chronisch entzündliche Darmerkrankungen (DACED) for financial support (to T.E.A). Finally, we thank Karina Greve and the IKMB NGS lab for excellent technical support.

\section{Author contributions}

H.T. and T.E.A. contributed equally to this work. L.M. and F.G. designed, performed, and analyzed most experiments and helped prepare the manuscript together with T.G. J.S., I.R. L.N., G.W.H., B.R., K.K., N.P., P.T., R.H., D.H., M.S., C.F., R.R.G., and B.E. G.O. and S.S. provided pathology expertise. M.K. helped with LC-MS/MS methodology. Q.R. and C.B. provided an experimental tool. R.K., M.E., and H.Z. provided access to clinical samples. F.S., P.R., H.Z., A.R.M., I.T., G.W., and A.K. helped develop the project. H.T. and T.E.A coordinated the project and prepared the manuscript.

\section{Competing interests}

The authors declare no competing interests.

\section{Additional information}

Supplementary information is available for this paper at https://doi.org/10.1038/s41467 020-15646-6.

Correspondence and requests for materials should be addressed to T.E.A

Peer review information Nature Communications thanks Vidya Velagapudi and the other anonymous reviewer(s) for their contribution to the peer review of this work. Peer reviewer reports are available.

Reprints and permission information is available at http://www.nature.com/reprints

Publisher's note Springer Nature remains neutral with regard to jurisdictional claims in published maps and institutional affiliations. 
(c) (i) Open Access This article is licensed under a Creative Commons Attribution 4.0 International License, which permits use, sharing, adaptation, distribution and reproduction in any medium or format, as long as you give appropriate credit to the original author(s) and the source, provide a link to the Creative Commons license, and indicate if changes were made. The images or other third party material in this article are included in the article's Creative Commons license, unless indicated otherwise in a credit line to the material. If material is not included in the article's Creative Commons license and your intended use is not permitted by statutory regulation or exceeds the permitted use, you will need to obtain permission directly from the copyright holder. To view a copy of this license, visit http://creativecommons.org/ licenses/by/4.0/.

(C) The Author(s) 2020 